\title{
Gravitational radiation from the classical spinning double copy
}

\author{
Jingping Li and Siddharth G. Prabhu \\ Physics Department, Yale University, New Haven, Connecticut 06520, USA
}

(Received 29 March 2018; published 29 May 2018)

\begin{abstract}
We establish a correspondence between perturbative classical gluon and gravitational radiation emitted by spinning sources, to linear order in spin. This is an extension of the nonspinning classical perturbative double copy and uses the same color-to-kinematic replacements. The gravitational theory has a scalar (dilaton) and a 2-form field (the Kalb-Ramond axion) in addition to the graviton. In a preceding paper [W. D. Goldberger et al., Phys. Rev. D 97, 105018 (2018)], we computed axion radiation in the gravitational theory to show that the correspondence fixes its action. Here, we present complete details of the gravitational computation. In particular, we also calculate the graviton and dilaton amplitudes in this theory and find that they precisely match with the predictions of the double copy. This constitutes a nontrivial check of the classical double copy correspondence and brings us closer to the goal of simplifying the calculation of gravitational wave observables for astrophysically relevant sources.
\end{abstract}

DOI: 10.1103/PhysRevD.97.105019

\section{INTRODUCTION}

Einstein's theory of general relativity, one of the most beautiful triumphs of modern physics, describes classical gravity to the best of our knowledge. However, the computational effort required to solve Einstein's equations, even perturbatively, is significant. On the other hand, in recent years, we have seen a series of remarkable developments in the study of perturbative scattering amplitudes in quantum field theory with both theoretical and practical significance. One could ask whether these methods are useful for the problem of obtaining solutions of classical gravity.

A recent promising approach in this direction relies on an idea first discovered in the context of quantum scattering amplitudes in gauge and gravity theories by Bern, Carrasco, and Johansson (BCJ) [1-3]. Numerators of gauge theory Feynman diagrams factorize into color factors (arising from the gauge group) and kinematic factors (made up of velocities, polarizations, etc.). Simply put, the BCJ prescription is to write the gauge theory amplitude in a certain form and replace every color factor with its kinematic factor counterpart. This procedure then gives the corresponding gravity amplitude. This $\mathrm{BCJ}$ double copy was, in turn, motivated by the closed string-open string amplitude relations due to Kawai, Lewellen, and Tye (KLT) [4]. KLT showed that the integrands of closed tree-level

Published by the American Physical Society under the terms of the Creative Commons Attribution 4.0 International license. Further distribution of this work must maintain attribution to the author(s) and the published article's title, journal citation, and DOI. Funded by SCOAP. string amplitudes factorize into open string ones. In the field theory limit, these express gravity tree amplitudes as a product of two corresponding gauge theory tree amplitudes. The BCJ double copy includes the field theory limit of the KLT relations as a special case. It has been proven for all tree-level scattering amplitudes [3] and there is increasing evidence at the loop level in various settings [5]. See [6] for a recent review.

The question of whether the double copy extends to classical solutions in gauge theory and general relativity was first raised in [7]. Their method of obtaining solutions of general relativity in the Kerr-Schild gauge was extended and studied in more detail in [8-10]. The BCJ double copy allows for the calculation of precision observables in gravity that were previously not amenable to a direct computation, by replacing such a calculation with the analogous gauge theory computation. Can such an idea be used to simplify the perturbative expansion of the equations of general relativity and reduce the computational effort required for gravitational wave calculations? ${ }^{1}$

Goldberger and Ridgway probed this question [14] by starting with a system of well-separated point color charges coupled to the Yang-Mills field. They calculated the Yang-Mills radiation that the sources generate, by selfconsistently solving the equations of motion for the sources and the field perturbatively. Remarkably, they found that a set of simple color-to-kinematic replacements produces gravitational radiation emitted by an analogous system of

\footnotetext{
${ }^{1}$ It is to be noted that an effective field theory approach to tackle the binary inspiral problem was introduced in [11] and extended to include spinning sources in [12]. A recent comprehensive review can be found in [13].
} 
point masses. It was shown in [15] that these color-tokinematic replacement rules can also be used to generate Yang-Mills radiation from scalar radiation, thereby completing a two-fold classical double copy for leading order radiation. This classical perturbative double copy was extended to radiation from sources in time-dependent orbits in [16], such as the bound orbits relevant for gravitational wave detection [17]. Reference [18] showed that EinsteinYang-Mills radiation can be obtained from Yang-Mills scalar radiation. Another approach to generate spacetime perturbatively, that is inspired by the double copy, can be found in [19].

In this paper, we complete the extension of the perturbative classical double copy to the case of radiation from spinning sources started in [20]. Our goal is to compute the gravitational radiation emitted from a system of spinning sources moving on general time-dependent trajectories, that satisfy the equations of motion, in $d$ dimensions. The motion of extended objects under a gravitational field has been approached through a variety of ways [21-25]. The formalism we use to describe spinning objects is detailed in the appendixes of [20] and is equivalent to the one used in $[12,26]$, in the context of effective field theories for extended gravitational sources.

Instead of attempting to solve Einstein's equations with spinning sources, we look to utilize the classical double copy [14]. To this end, we consider, instead, a system of point colored charges, with color variable $c_{a}(\tau)$ [27], that couple to the Yang-Mills field. ${ }^{2}$ Each point charge possesses a spin angular momentum $S^{\mu \nu}(\tau)$ which couples to the Yang-Mills field via a chromomagnetic spin dipole coupling

$$
S_{\mathrm{int}}=\frac{g_{s} \kappa}{2} \int d \tau c_{a} S^{\mu \nu} F_{\mu \nu}^{a}
$$

with coupling strength $\kappa$ and $\tau$ the worldline coordinate. We let the particles evolve self-consistently under their equations of motion and compute, to linear order in spin, the amplitude of Yang-Mills radiation $\mathcal{A}_{a}^{\mu}(k)$ that they generate. We then employ the simple color-to-kinematics substitutions [14,16] to get a double copy radiation amplitude $\mathcal{A}^{\mu \nu}(k)$,

$$
\epsilon_{\mu}^{* a}(k) \mathcal{A}_{a}^{\mu}(k) \mapsto \epsilon_{\mu}^{*}(k) \tilde{\epsilon}_{\nu}^{*}(k) \mathcal{A}^{\mu \nu}(k) .
$$

Consistency of the double copy amplitude $\mathcal{A}^{\mu \nu}(k)$ with gravitational Ward identities sets the chromomagnetic dipole coupling strength $\kappa$ for each particle to be the same constant $\kappa=-1$. The double copy amplitude $\mathcal{A}^{\mu \nu}(k)$ can, in general, be decomposed into its antisymmetric,

\footnotetext{
${ }^{2}$ Finite size corrections are systematically accounted for by including higher order terms in an effective field theory framework; see [11].
}

symmetric-traceless, and trace components. The corresponding radiation fields are also expected by decomposing products of vector irreducible representations of the massless little group $S O(d-2)$,

$$
\begin{gathered}
n \otimes n=1 \oplus \frac{n(n+1)}{2}-1 \oplus \frac{n(n-1)}{2}, \\
A_{\mu} \otimes A_{\nu}=\phi \oplus h_{\mu \nu} \oplus B_{\mu \nu},
\end{gathered}
$$

where $\phi$ is a scalar (dilaton), $h_{\mu \nu}$ the graviton, and $B_{\mu \nu}$ the Kalb-Ramond axion [28]. ${ }^{3}$

In the case of nonspinning sources, the double copied field is symmetric, thereby implying the field content of the gravitational theory to be $\left(h_{\mu \nu}, \phi\right)$. This can be understood by noting that one cannot write down a linear interaction of nonspinning particles with the axion field. Alternately, in this case, gravitational radiation can be seen as arising as a two-fold double copy of the biadjoint scalar radiation [15]. The latter theory enjoys a $G \times \tilde{G}$ global symmetry and is invariant under the exchange of these two groups. The color-kinematic substitution rules, which take an adjoint index of each group to a Lorentz index, treat both adjoint indices corresponding to these two groups symmetrically. Hence, the resulting gravitational radiation is symmetric under the exchange of the Lorentz indices. The action of the gravitational theory was shown to be [14]

$$
\begin{aligned}
S= & -2 m_{\mathrm{Pl}}^{d-2} \int d^{d} x \sqrt{g}\left[R-(d-2) g^{\mu \nu} \partial_{\mu} \phi \partial_{\nu} \phi\right] \\
& -\sum_{\alpha} m_{\alpha} \int d \tau e^{\phi} .
\end{aligned}
$$

For spinning sources, we expect the field content of the gravitational theory to be $\left(h_{\mu \nu}, B_{\mu \nu}, \phi\right)$. Decomposing the double copy amplitude lands us at graviton, dilaton, and axion radiation in this theory. We write down the most general action with two derivatives using diffeomorphism invariance and 2-form gauge invariance. Consistency with the double copy fixes the action to be

$$
\begin{aligned}
S_{g}= & -2 m_{\mathrm{Pl}}^{d-2} \int d^{d} x \sqrt{g}\left[R-(d-2) g^{\mu \nu} \partial_{\mu} \phi \partial_{\nu} \phi\right. \\
& \left.+\frac{1}{12} e^{-4 \phi} H_{\mu \nu \sigma} H^{\mu \nu \sigma}\right],
\end{aligned}
$$

where $H_{\mu \nu \sigma}=(d B)_{\mu \nu \sigma}$ is the field strength of the 2-form. This action also describes the BCJ double copy of pure gluons [3] (see also [19]) and appears in the low energy effective action of oriented closed strings. Compared to the nonspinning case, the spinning sources have an additional interaction, namely, that with the axion field given by

\footnotetext{
${ }^{3}$ To be explicit, we refer to the 2-form field $B_{\mu \nu}$ in any dimension as the axion.
} 


$$
S_{H S}=\frac{1}{4} \int d x^{\mu} H_{\mu \nu \sigma} S^{\nu \sigma} e^{-2 \phi}
$$

We note that this action differs from the one in [20] as the "string frame" metric $\tilde{g}_{\mu \nu}=g_{\mu \nu} e^{2 \phi}$ was used to define spin there, as opposed to the ordinary metric $g_{\mu \nu}$ used in this paper (for more details, refer to Sec. IV).

The rest of the paper is organized as follows. In Sec. II, we review the computation of classical gluon radiation from a system of several spinning sources to leading order in spin that was obtained in [20]. We obtain the double copy of this radiation amplitude in Sec. III and decompose it into radiation in the graviton, dilaton, and axion channels. In Sec. IV, we calculate the corresponding radiation amplitudes emitted by a collection of several spinning masses in the gravitational theory given by Eqs. (6) and (7). We discuss our results and further questions raised in Sec. V.

\section{YANG-MILLS RADIATION}

We begin by reviewing the calculation of Yang-Mills radiation emitted by a classical system of several spinning colored particles in $d$ dimensions, presented in [20]. For each particle, with worldline coordinate $s$, the degrees of freedom are a worldline trajectory $x^{\mu}(s)$, a spin angular momentum $S^{\mu \nu}(s)$, and a color charge $c^{a}(s)$ [27] transforming in the adjoint representation of the gauge group $G$. We first present some details of the spinning formalism of [20] that are needed to describe the interactions of such a system with a gauge field. ${ }^{4}$

In order to describe the spin degree of freedom, we endow each worldline with an orthonormal reference frame $e_{\mu}^{I}(s)$ [24]. The spin $S^{I J}(s)$ is then introduced as the variable conjugate to the angular velocity

$$
\Omega^{I J} \equiv \eta^{\mu \nu} e_{\mu}^{I} \frac{d}{d s} e_{\nu}^{J}=-\Omega^{J I},
$$

whereas the momentum $p_{I}(s)$ is conjugate to $x_{I}(s)$. In $d$ dimensions, the number of spatial rotational degrees of freedom is $\frac{1}{2}(d-1)(d-2)$. Hence, we need to impose a constraint on the spin angular momentum to get this physical number of degrees of freedom. Following $[24,29]$, this can be done in a number of different ways. We choose to impose the constraint that the spin is transverse to the momentum,

$$
S^{\mu \nu} p_{\nu}=0 .
$$

We also introduce an einbein $e$ that enforces worldline reparametrization invariance $[e(s) d s$ is invariant under

\footnotetext{
${ }^{4}$ We use the conventions $D_{\mu}=\partial_{\mu}+i g_{s} A_{\mu}^{a} T^{a},\left[T^{a}, T^{b}\right]=i f^{a b c} T^{c}$, and $\left(T_{\text {adj }}^{a}\right)_{c}^{b}=-i f_{a b c}$.
}

$s \mapsto s^{\prime}(s)$ ], and a Lagrange multiplier $\lambda_{I}$ that enforces the spin constraint above.

Each particle is described by the action

$$
\begin{aligned}
S_{p p}= & -\int d x^{\mu} e_{\mu}^{I} p_{I}+\frac{1}{2} \int d s e\left(p_{I} p^{I}-m^{2}(S)+\cdots\right) \\
& +\frac{1}{2} \int d s S^{I J} \Omega_{I J}+\int d s e \lambda_{I} S^{I J} p_{J} \\
& -g_{s} \int d x^{\mu} c_{a} A_{\mu}^{a}+\frac{g_{s} \kappa}{2} \int d s e e_{a} S^{\mu \nu} F_{\mu \nu}^{a},
\end{aligned}
$$

where the first line has all the terms that describe a free particle, and the second line contains the interaction terms of the particle with the gauge field. Here, $g_{s}$ is the YangMills coupling constant, and $\kappa$ is the spin dipole coupling constant. This action, together with the usual Yang-Mills action in the bulk, constitutes the complete action for the system of particles interacting with a gauge field.

The resulting equations of motion are the following. Varying the action with respect to the gauge field, we have the usual Yang-Mills field equations,

$$
D_{\nu} F_{a}^{\nu \mu}(x)=g_{s} J_{a}^{\mu}(x),
$$

with the color current generated by the particles given by

$$
\begin{aligned}
J_{a}^{\mu}(x) \equiv & -\frac{1}{g_{s}} \frac{\delta}{\delta A_{\mu}^{a}(x)} S_{p p} \\
= & \sum_{\alpha} \int d x_{\alpha}^{\mu} c_{\alpha}^{a} \frac{\delta^{d}\left(x-x_{\alpha}\left(s_{\alpha}\right)\right)}{\sqrt{g}} \\
& -\kappa_{\alpha} \int d s_{\alpha} e_{\alpha} S^{\mu \nu} D_{\nu}\left[c_{\alpha}^{a} \frac{\delta^{d}\left(x-x_{\alpha}\left(s_{\alpha}\right)\right)}{\sqrt{g}}\right] .
\end{aligned}
$$

Here, the sum runs over all the particles, indexed by $\alpha$.

Imposing current conservation covariantly, $D_{\mu} J_{a}^{\mu}=0$, gives the equation of motion in color space,

$$
\left(\dot{x}_{\alpha} \cdot D\right) c_{\alpha}^{a}=\frac{i \kappa_{\alpha} g_{s}}{2} e_{\alpha} S_{\alpha}^{\mu \nu}\left[F_{\mu \nu}, c_{\alpha}\right]^{a} .
$$

The energy-momentum tensor for a single spinning particle comes out to be

$$
\begin{aligned}
T_{p p}^{\mu \nu}(x) \equiv & -\frac{2}{\sqrt{g}} \frac{\delta}{\delta g_{\mu \nu}(x)} S_{p p}=\int d x^{(\mu} p^{\nu)} \delta(x-x(s)) \\
& +\int d x^{(\mu} S^{\nu) \sigma} \partial_{\sigma} \delta(x-x(s)) \\
& -\kappa g_{s} \int d s \frac{\delta(x-x(s))}{\sqrt{g}} c_{a} F^{a}{ }_{\sigma}{ }^{(\mu} S^{\nu) \sigma},
\end{aligned}
$$

where the brackets () indicate symmetrization of the corresponding indices. The integral of the divergence of conserved currents with arbitrary support $X$ should vanish 
on shell: $\left.\int d^{d} x \sqrt{g} X_{\nu} \nabla_{\mu}\left(T_{\mathrm{YM}}^{\mu \nu}+T_{p p}^{\mu \nu}\right)\right|_{\text {on-shell }}=0$. This leads to the equations of motion for the momentum and the spin,

$$
\begin{gathered}
\frac{d}{d s} p_{\alpha}^{\mu}=g_{s} c_{\alpha}^{a} F_{a}^{\mu \nu} \dot{x}_{\alpha \nu}-\frac{\kappa_{\alpha} g_{s} e_{\alpha}}{2} S_{\alpha}^{\rho \sigma} c_{\alpha}^{a} D^{\mu} F_{\rho \sigma}^{a}, \\
\frac{d}{d s} S_{\alpha}^{\mu \nu}=\dot{x}_{\alpha}^{\nu} p_{\alpha}^{\mu}-\dot{x}_{\alpha}^{\mu} p_{\alpha}^{\nu}-\kappa_{\alpha} g_{s} e_{\alpha} c_{\alpha}^{a} F_{\sigma}^{a \mu} S_{\alpha}^{\nu \sigma}+\kappa_{\alpha} g_{s} e_{\alpha} c_{\alpha}^{a} F_{\sigma}^{a \nu} S_{\alpha}^{\mu \sigma} .
\end{gathered}
$$

The motion of the particles is thus described by the system of equations (13), (15), and (16), subject to the constraint Eq. (9). Alternately, these equations of motion can also be obtained by varying the action with respect to $\left(x^{\mu}, e_{\mu}^{I}, e, p_{I}, S^{I J}, \lambda_{I}\right)$. The constants of the motion are $c_{a} c^{a}$, $S_{\mu \nu} S^{\mu \nu}$, and $m^{2}=p_{\mu} p^{\mu}+g_{s} \kappa c_{a} S^{\mu \nu} F_{\mu \nu}^{a}$.

From the invariance of the spin constraint,

$$
\frac{d}{d s}\left(S^{\mu \nu} p_{\nu}\right)=0
$$

we can solve for the velocity $v^{\mu} \equiv \dot{x}^{\mu}$ in terms of the other variables. In the following, we use reparametrization freedom to choose $e_{\alpha}$ such that $s_{\alpha}=\tau_{\alpha}$, the proper time for each particle, whereby $p_{\alpha}^{\mu} \simeq v_{\alpha}^{\mu}$ up to $O\left(S^{0}\right)$.

In the Lorenz gauge, $\partial_{\mu} A_{a}^{\mu}=0$, the Yang-Mills field equations (11) take the form

$\square A_{a}^{\mu} \equiv g_{s} \tilde{J}_{a}^{\mu}(x)=g_{s} J_{a}^{\mu}+g_{s} f^{a b c} A_{\nu}^{b}\left(\partial^{\nu} A_{c}^{\mu}-F_{c}^{\mu \nu}\right)$,

defining the source current $\tilde{J}_{a}^{\mu}(x)$, which includes contributions from both the point sources as well as the field configuration. It is conserved, $\partial_{\mu} \tilde{J}_{a}^{\mu}(x)=0$, and related to observables measured at null infinity. The specific relation between the radiation field at null infinity and the source current in momentum space $\tilde{J}_{a}^{\mu}(k)=\int d^{d} x e^{i k \cdot x} \tilde{J}_{a}^{\mu}(x)$ depends on the dimension $d$. For example, in $d=4$ dimensions, the radiation field is given by

$$
\lim _{r \rightarrow \infty} A_{a}^{\mu}(x)=\frac{g_{s}}{4 \pi r} \int \frac{d \omega}{2 \pi} e^{-i \omega t} \tilde{J}_{a}^{\mu}(k),
$$

with $k^{\mu}=(\omega, \vec{k})=\omega(1, \vec{x} / r)$. In any dimensions, the total energy-momentum radiated out to infinity in polarization channel $r$ is given by

$$
\Delta P_{\mathrm{r}}^{\mu}=\int_{k}(2 \pi) \theta\left(k^{0}\right) \delta\left(k^{2}\right) k^{\mu}\left|\epsilon_{\mathrm{r}, \mathcal{L}}^{* a}(k) g_{s} \tilde{J}_{a}^{\nu}(k)\right|^{2},
$$

with $\epsilon_{\mathrm{r}, \mu}^{a}(k)$ being gluon polarization vectors. These are normalized as $\epsilon_{\mathrm{r}}^{* a}(k) \cdot \epsilon_{\mathrm{r}^{\prime}}^{b}(k)=-\delta_{a b} \delta_{\mathrm{rr}^{\prime}}$ and satisfy the gauge condition $k \cdot \epsilon_{\mathrm{r}}^{a}(k)=0$. (The polarization indices do not play any role in our calculations, so they will be suppressed from now on.) Suitable integrals of the
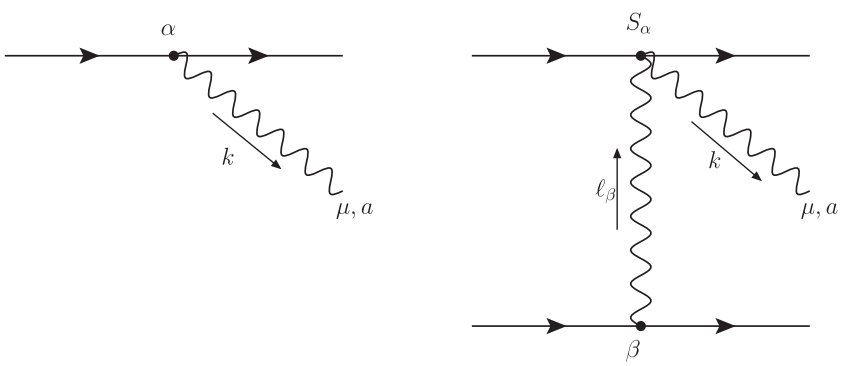

(a)

(c)



(b)

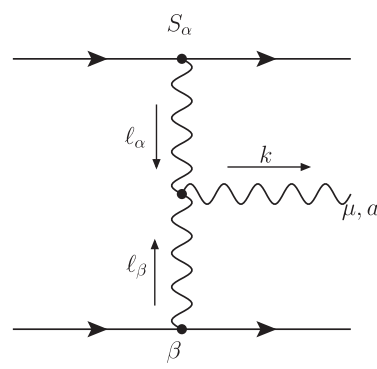

(d)

FIG. 1. Feynman diagrams for the perturbative expansion of $\tilde{J}_{a}^{\mu}(k)$ up to order $\mathcal{O}\left(g_{s}^{2}\right)$ and to linear order in spin. (a) Contribution to the spin-independent color current due to the equations of motion. (b)-(d) Contributions from a single insertion of the spin-dependent color current.

momentum space source current $\tilde{J}_{a}^{\mu}(k)$ thus produce physical observables at null infinity. Hence, in what follows, our object of interest is $\tilde{J}_{a}^{\mu}(k)$. We compute it perturbatively in the Yang-Mills coupling constant, ${ }^{5}$ and to linear order in spin, by consistently solving the system of equations for the particles and the field.

In the following, we employ the same notation as in [20],

$$
\begin{gathered}
S_{\alpha}^{\mu \nu} p_{\nu} \equiv\left(S_{\alpha} \wedge p\right)^{\mu}, \\
k_{\mu} S_{\alpha}^{\mu \nu} p_{\nu} \equiv(k \wedge p)_{\alpha},
\end{gathered}
$$

to denote contractions of the spin angular momentum with any Lorentz vectors $k$ and $p$. We also use $\mathcal{O}(\ldots)$ notation to denote contributions at a particular order.

The leading order current can be seen as the contributions of the Feynman diagrams in Figs. 1(a) and 1(b) to lowest order in the coupling constant. Following the results in [16], we can work with particles traveling along general time-dependent (possibly bound) orbits, say $x_{\alpha}^{\mu}\left(\tau_{\alpha}\right), p_{\alpha}^{\mu}\left(\tau_{\alpha}\right)$, $c_{\alpha}^{a}\left(\tau_{\alpha}\right)$, and $S_{\alpha}^{\mu \nu}\left(\tau_{\alpha}\right)$. In the following, we drop the explicit dependence on $\tau$, so that, for example, $x_{\alpha}^{\mu} \equiv x_{\alpha}^{\mu}\left(\tau_{\alpha}\right)$. Then to all orders in perturbation, the contribution from the sum of these diagrams can be written as

\footnotetext{
${ }^{5}$ See [14-16] for a detailed discussion of the explicit small expansion parameter that suppresses higher order contributions.
} 


$$
\left.\tilde{J}_{a}^{\mu}(k)\right|_{\text {Fig. } 1(\mathrm{a})+(\mathrm{b})}=\sum_{\alpha} \int d \tau_{\alpha} e^{i k \cdot x_{\alpha}} c_{\alpha}^{a}\left[v_{\alpha}^{\mu}+i \kappa_{\alpha}\left(S_{\alpha} \wedge k\right)^{\mu}\right]
$$

At leading order, this gives rise to the field ${ }^{6}$

$$
\left.A_{a}^{\mu}(x)\right|_{\mathcal{O}\left(g_{s}^{1}\right)}=\left.g_{s} \int \frac{d^{d} \ell}{(2 \pi)^{d}} \frac{e^{-i \ell \cdot x}}{\ell^{2}} \tilde{J}_{a}^{\mu}(\ell)\right|_{\mathcal{O}\left(g_{s}^{1}\right)}=g_{s} \sum_{\alpha} \int d \tau_{\alpha} \frac{d^{d} \ell}{(2 \pi)^{d}} \frac{e^{-i \ell \cdot\left(x-x_{\alpha}\right)}}{\ell^{2}} c_{\alpha}^{a}\left[p_{\alpha}^{\mu}+i \kappa_{\alpha}\left(S_{\alpha} \wedge \ell\right)^{\mu}\right] .
$$

This lowest order field then induces corrections in color, position, momentum, and spin of the particles, which causes the particle to radiate at the next order in perturbation.

The first contribution to radiation comes from gluons emitted directly by the particles. This is the $\mathcal{O}\left(g_{s}^{2}\right)$ contribution to Eq. (23), given by

$$
\begin{gathered}
\left.\tilde{J}_{a}^{\mu}(k)\right|_{\text {Fig. 1(a) }+(\mathrm{b}) ; \mathcal{O}\left(g_{s}^{2}, S^{1}\right)}=\left.\sum_{\alpha} \int d \tau_{\alpha} e^{i k \cdot x_{\alpha}} \frac{i}{k \cdot v_{\alpha}}\left[\dot{c}_{\alpha}^{a} v_{\alpha}^{\mu}+c_{\alpha}^{a}\left\{\dot{v}_{\alpha}^{\mu}-\frac{k \cdot \dot{v}_{\alpha}}{k \cdot v_{\alpha}} v_{\alpha}^{\mu}\right\}\right]\right|_{\mathcal{O}\left(g_{s}^{2}, S^{1}\right)} \\
\quad-\left.\sum_{\alpha} \kappa_{\alpha} \int d \tau_{\alpha} e^{i k \cdot x_{\alpha}} \frac{1}{k \cdot v_{\alpha}}\left[\dot{c}_{\alpha}^{a}\left(S_{\alpha} \wedge k\right)+c_{\alpha}^{a}\left\{\left(\dot{S}_{\alpha} \wedge k\right)-\frac{k \cdot \dot{v}_{\alpha}}{k \cdot v_{\alpha}}\left(S_{\alpha} \wedge k\right)^{\mu}\right\}\right]\right|_{\mathcal{O}\left(g_{s}^{2}, S^{1}\right)},
\end{gathered}
$$

where the first line corresponds to inserting the spin-dependent solutions [20], while the second line corresponds to inserting spin-independent solutions [14]. The explicit expressions are given by Eqs. (A1) and (A2) in Appendix A 1, where we have introduced an integration measure over worldline parameters and momenta given by

$$
d \mu_{\alpha \beta}(k) \equiv d \tau_{\alpha} d \tau_{\beta}\left[\frac{d^{d} \ell_{\alpha}}{(2 \pi)^{d}} \frac{e^{i \ell_{\alpha} \cdot x_{\alpha}}}{\ell_{\alpha}^{2}}\right]\left[\frac{d^{d} \ell_{\beta}}{(2 \pi)^{d}} \frac{e^{i \ell_{\beta} \cdot x_{\beta}}}{\ell_{\beta}^{2}}\right](2 \pi)^{d} \delta^{d}\left(k-\ell_{\alpha}-\ell_{\beta}\right) .
$$

At this order $O\left(g_{s}^{2}\right)$ in perturbation, there are two contributions from diagrams without deflections in the particle trajectories. The first of these is from Fig. 1(c),

$$
\left.\tilde{J}_{a}^{\mu}(k)\right|_{\text {Fig. } 1(\mathrm{c}) ; \mathcal{O}\left(g_{s}^{2}, S^{1}\right)}=i g_{s}^{2} \sum_{\substack{\alpha, \beta \\ \alpha \neq \beta}} \int d \mu_{\alpha \beta}(k)\left[c_{\alpha}, c_{\beta}\right]^{a} \ell_{\alpha}^{2}\left(S_{\alpha} \wedge p_{\beta}\right)^{\mu} .
$$

The second of the zero deflection contributions is from the diagram with the triple vertex in Fig. 1(d),

$\left.\tilde{J}_{a}^{\mu}(k)\right|_{\left.\text {Fig. 1(d);O(g } g_{s}^{2}, S^{1}\right)}=-i g_{s}^{2} \sum_{\substack{\alpha, \beta \\ \alpha \neq \beta}} \int d \mu_{\alpha \beta}(k)\left[c_{\alpha}, c_{\beta}\right]^{a}\left[2\left(k \cdot p_{\beta}\right)\left(S_{\alpha} \wedge \ell_{\alpha}\right)^{\mu}+\left(\ell_{\alpha} \wedge p_{\beta}\right)_{\alpha}\left(\ell_{\alpha}-\ell_{\beta}\right)^{\mu}+2\left(\ell_{\alpha} \wedge \ell_{\beta}\right)_{\alpha} p_{\beta}^{\mu}\right]$.

We can write down the total expression for the leading order radiation (as written in [20]) coming from spinning particles in general orbits consistent with the equations of motion. The result is a sum of two color structures:

$$
\left.\tilde{J}_{a}^{\mu}(k)\right|_{\mathcal{O}\left(g_{s}^{2}, S^{1}\right)}=i g_{s}^{2} \sum_{\substack{\alpha, \beta \\ \alpha \neq \beta}} \int d \mu_{\alpha \beta}(k)\left[\left(c_{\alpha} \cdot c_{\beta}\right) c_{\alpha}^{a} \mathcal{A}_{s}^{\mu}+\left[c_{\alpha}, c_{\beta}\right]^{a} \mathcal{A}_{\mathrm{adj}}^{\mu}\right]
$$

with

\footnotetext{
${ }^{6}$ As we are in a classical setup, our propagators, here and in the rest of the paper, are assumed to be defined with retarded boundary conditions $1 / k^{2}=1 /\left(\left(k^{0}+i \epsilon\right)^{2}-\vec{k}^{2}\right)$ and $1 / k \cdot p=1 /(k \cdot p+i \epsilon)$.
} 


$$
\begin{aligned}
\mathcal{A}_{\mathrm{adj}}^{\mu} \equiv & \kappa_{\alpha}\left[\left(\ell_{\alpha} \wedge p_{\beta}\right)_{\alpha}\left(\ell_{\beta}-\ell_{\alpha}\right)^{\mu}-\frac{\ell_{\alpha}^{2}}{k \cdot p_{\alpha}}\left(\ell_{\beta} \wedge p_{\beta}\right)_{\alpha} p_{\alpha}^{\mu}-\frac{\ell_{\beta}^{2}}{k \cdot p_{\beta}}\left(\ell_{\alpha} \wedge p_{\beta}\right)_{\alpha} p_{\beta}^{\mu}+\ell_{\alpha}^{2}\left(S_{\alpha} \wedge p_{\beta}\right)^{\mu}\right] \\
& -2 \kappa_{\alpha}\left(k \cdot p_{\beta}\right)\left[\left(S_{\alpha} \wedge \ell_{\alpha}\right)^{\mu}-\frac{\left(k \wedge \ell_{\alpha}\right)_{\alpha}}{k \cdot p_{\beta}} p_{\beta}^{\mu}\right]-\kappa_{\alpha} \frac{\ell_{\alpha}^{2}}{k \cdot p_{\alpha}}\left(p_{\alpha} \cdot p_{\beta}\right)\left(S_{\alpha} \wedge k\right)^{\mu},
\end{aligned}
$$

and

$$
\begin{aligned}
\mathcal{A}_{s}^{\mu} \equiv & \frac{\left(1+\kappa_{\alpha}\right)^{2}}{m_{\alpha}^{2}} \ell_{\alpha}^{2}\left[\left(k \cdot p_{\alpha}\right)\left\{\left(S_{\alpha} \wedge p_{\beta}\right)^{\mu}-\frac{\left(k \wedge p_{\beta}\right)_{\alpha}}{k \cdot p_{\alpha}} p_{\alpha}^{\mu}\right\}+\left(p_{\alpha} \cdot p_{\beta}\right)\left\{\left(S_{\alpha} \wedge \ell_{\beta}\right)^{\mu}-\frac{\left(k \wedge \ell_{\beta}\right)_{\alpha}}{k \cdot p_{\alpha}} p_{\alpha}^{\mu}\right\}\right] \\
& -\kappa_{\beta} \ell_{\alpha}^{2}\left[\left(S_{\beta} \wedge \ell_{\beta}\right)^{\mu}-\frac{\left(k \wedge \ell_{\beta}\right)_{\beta}}{k \cdot p_{\alpha}} p_{\alpha}^{\mu}\right] \\
& +\kappa_{\alpha}^{2} \frac{\ell_{\alpha}^{2}}{k \cdot p_{\alpha}}\left[\left(k \cdot p_{\beta}\right)\left\{\left(S_{\alpha} \wedge \ell_{\beta}\right)^{\mu}-\frac{\left(k \wedge \ell_{\beta}\right)_{\alpha}}{k \cdot p_{\beta}} p_{\beta}^{\mu}\right\}-\left(k \cdot \ell_{\beta}\right)\left\{\left(S_{\alpha} \wedge p_{\beta}\right)^{\mu}-\frac{\left(k \wedge p_{\beta}\right)_{\alpha}}{k \cdot p_{\beta}} \ell_{\beta}^{\mu}\right\}\right] \\
& +\kappa_{\alpha} \frac{\ell_{\alpha}^{2}}{k \cdot p_{\alpha}}\left[\left(\ell_{\beta} \wedge p_{\beta}\right)_{\alpha}\left\{\ell_{\beta}^{\mu}-\frac{k \cdot \ell_{\beta}}{k \cdot p_{\alpha}} p_{\alpha}^{\mu}\right\}+\left(k \cdot p_{\beta}\right)\left(S_{\alpha} \wedge k\right)^{\mu}\right]+\kappa_{\beta} \frac{\ell_{\alpha}^{2}}{k \cdot p_{\alpha}}\left(\ell_{\beta} \wedge p_{\alpha}\right)_{\beta}\left[\ell_{\beta}^{\mu}-\frac{k \cdot \ell_{\beta}}{k \cdot p_{\alpha}} p_{\alpha}^{\mu}\right] \\
& -\kappa_{\alpha} \frac{\ell_{\alpha}^{2}}{\left(k \cdot p_{\alpha}\right)^{2}}\left(p_{\alpha} \cdot p_{\beta}\right)\left(k \cdot \ell_{\beta}\right)\left(S_{\alpha} \wedge k\right)^{\mu} .
\end{aligned}
$$

It is easy to check that this result satisfies the Ward identity $k_{\mu} \tilde{J}_{a}^{\mu}(k)=0$ even off shell.

\section{DOUBLE COPY}

As in the spinless case $[14,16]$, we transform the YangMills radiation by the following set of color-kinematic substitution rules:

$$
\begin{gathered}
c_{\alpha}^{a}(\tau) \mapsto p_{\alpha}^{\mu}(\tau), \\
{\left[c_{\alpha}(\tau), c_{\beta}(\tau)\right]^{a} \mapsto \Gamma^{\mu \nu \rho}\left(-k, \ell_{\alpha}, \ell_{\beta}\right) p_{\nu \alpha}(\tau) p_{\rho \beta}(\tau),}
\end{gathered}
$$

where $\Gamma^{\mu \nu \rho}\left(-k, \ell_{\alpha}, \ell_{\beta}\right)$ is the kinematic part of the 3-point gluon vertex Feynman rule, defined as

$$
\begin{aligned}
& \Gamma^{\mu \nu \rho}\left(-k, \ell_{\alpha}, \ell_{\beta}\right) \\
& \quad \equiv \frac{1}{2}\left[\left(\ell_{\beta}-\ell_{\alpha}\right)^{\mu} \eta^{\nu \rho}+\left(\ell_{\alpha}+k\right)^{\rho} \eta^{\mu \nu}-\left(\ell_{\beta}+k\right)^{\nu} \eta^{\mu \rho}\right] .
\end{aligned}
$$

We also identify the respective coupling constants,

$$
g_{s} \mapsto \frac{1}{2 m_{\mathrm{Pl}}^{(d-2) / 2}} \equiv \eta .
$$

In the nonspinning case, the momenta remained unchanged under the double copy $p_{\alpha}^{\mu}(\tau) \mapsto p_{\alpha}^{\mu}(\tau)$. Similarly, in the spinning case, we use the substitution $S_{\alpha}^{\mu \nu}(\tau) \mapsto S_{\alpha}^{\mu \nu}(\tau)$. We use the above substitution rules to transform the Yang-Mills radiation amplitude $\mathcal{A}_{a}^{\mu}(k)$, defined as $\mathcal{A}_{a}^{\mu}(k) \equiv$ $\left.g_{s} \tilde{J}_{a}^{\mu}(k)\right|_{k^{2}=0}$, and obtain the double copy radiation amplitude $\mathcal{A}^{\mu \nu}(k)$, with $k^{2}=0$, as

$$
\epsilon_{\mu}^{* a}(k) \mathcal{A}_{a}^{\mu}(k) \mapsto \epsilon_{\mu}^{*}(k) \tilde{\epsilon}_{\nu}^{*}(k) \mathcal{A}^{\mu \nu}(k),
$$

where the on-shell gluon polarization $\epsilon_{\mu}^{* a}(k)$ has been formally replaced by a product of on-shell independent polarizations $\epsilon_{\mu}^{*}(k) \tilde{\epsilon}_{\nu}^{*}(k)$. Thus, the double copy amplitude $\mathcal{A}^{\mu \nu}(k)$ is defined up to terms that vanish when dotted into these polarization vectors. Explicitly, it is given by

$\left.\mathcal{A}^{\mu \nu}(k)\right|_{\mathcal{O}\left(\eta^{3}, S^{1}\right)}=\frac{i}{8 m_{\mathrm{Pl}}^{(d-2) / 2}} \sum_{\substack{\alpha, \beta \\ \alpha \neq \beta}} \int d \mu_{\alpha \beta}(k)\left[\left(\frac{1}{2}\left(p_{\alpha} \cdot p_{\beta}\right)\left(\ell_{\beta}-\ell_{\alpha}\right)^{\nu}+\left(k \cdot p_{\beta}\right) p_{\alpha}^{\nu}-\left(k \cdot p_{\alpha}\right) p_{\beta}^{\nu}\right) \mathcal{A}_{\mathrm{adj}}^{\mu}-\left(p_{\alpha} \cdot p_{\beta}\right) p_{\alpha}^{\nu} \mathcal{A}_{s}^{\mu}\right]$,

where $\mathcal{A}_{\text {adj }}^{\mu}$ and $\mathcal{A}_{s}^{\mu}$ are given in Eqs. (30) and (31), respectively.

We see that $k_{\mu} \tilde{J}^{\mu}(k)=0$ automatically guarantees $k_{\mu} \mathcal{A}^{\mu \nu}(k)=0$ because the color-kinematic substitution rules do not affect this Lorentz index. For $\mathcal{A}^{\mu \nu}(k)$ to define the radiation amplitudes consistently in a gravitational theory, we also need it to satisfy the Ward identity in the second Lorentz index $k_{\nu} \mathcal{A}^{\mu \nu}(k)=0$. Unlike the 
nonspinning case, this now imposes an extra constraint on the Yang-Mills theory [20], namely, that

$$
\kappa_{\alpha}=-1 .
$$

Thus, while the Yang-Mills theory is consistent for any value of the chromomagnetic coupling constant $\kappa_{\alpha}$, we find that the double copy procedure only works when all the particles couple to the gauge field with this special value of the coupling constant. As was noted in [20], in $d=4$, this value corresponds to the so-called "natural" value [30,31] of the gyromagnetic ratio $g=2$. For this special value of the coupling constant, we can write the double copy amplitude as

$$
\begin{aligned}
& \left.\mathcal{A}^{\mu \nu}(k)\right|_{\mathcal{O}\left(\eta^{3}, S^{1}\right)}=-\frac{i}{8 m_{\mathrm{Pl}}^{3(d-2) / 2}} \sum_{\substack{\alpha, \beta \\
\alpha \neq \beta}} \int d \mu_{\alpha \beta}(k)\left[\frac { \ell _ { \alpha } ^ { 2 } ( p _ { \alpha } \cdot p _ { \beta } ) p _ { \alpha } ^ { \nu } } { k \cdot p _ { \alpha } } \left\{\frac { k \cdot \ell _ { \beta } } { k \cdot p _ { \alpha } } \left(\left(p_{\alpha} \cdot p_{\beta}\right)\left(S_{\alpha} \wedge k\right)^{\mu}+p_{\alpha}^{\mu}\left[\left(\ell_{\beta} \wedge p_{\beta}\right)_{\alpha}\right.\right.\right.\right. \\
& \left.\left.-\left(\ell_{\beta} \wedge p_{\alpha}\right)_{\beta}\right]\right)-\left(k \cdot \ell_{\beta}\right)\left(S_{\alpha} \wedge p_{\beta}\right)^{\mu}+\frac{1}{2}\left(\ell_{\beta}^{\mu}-\ell_{\alpha}^{\mu}\right)\left[\left(\ell_{\alpha} \wedge p_{\beta}\right)_{\alpha}+\left(\ell_{\beta} \wedge p_{\alpha}\right)_{\beta}\right]-p_{\alpha}^{\mu}\left(\ell_{\alpha} \wedge \ell_{\beta}\right)_{\beta} \\
& \left.-p_{\beta}^{\mu}\left(\ell_{\alpha} \wedge \ell_{\beta}\right)_{\alpha}-\left(k \cdot p_{\beta}\right)\left(S_{\alpha} \wedge \ell_{\alpha}\right)^{\mu}+\left(k \cdot p_{\alpha}\right)\left(S_{\beta} \wedge \ell_{\beta}\right)^{\mu}\right\}+\left\{p_{\alpha}^{\nu}\left(k \cdot p_{\beta}\right)-p_{\beta}^{\nu}\left(k \cdot p_{\alpha}\right)\right. \\
& \left.+\frac{1}{2}\left(\ell_{\beta}^{\nu}-\ell_{\alpha}^{\nu}\right)\left(p_{\alpha} \cdot p_{\beta}\right)\right\}\left\{-\frac{\ell_{\alpha}^{2}}{k \cdot p_{\alpha}}\left(\left(p_{\alpha} \cdot p_{\beta}\right)\left(S_{\alpha} \wedge k\right)^{\mu}-p_{\alpha}^{\mu}\left[\left(\ell_{\beta} \wedge p_{\alpha}\right)_{\beta}-\left(\ell_{\beta} \wedge p_{\beta}\right)_{\alpha}\right]\right)\right. \\
& \left.\left.+\ell_{\beta}^{\mu}\left[\left(\ell_{\beta} \wedge p_{\alpha}\right)_{\beta}+\left(\ell_{\alpha} \wedge p_{\beta}\right)_{\alpha}\right]-2 p_{\beta}^{\mu}\left(\ell_{\alpha} \wedge \ell_{\beta}\right)_{\alpha}+2\left(k \cdot p_{\alpha}\right)\left(S_{\beta} \wedge \ell_{\beta}\right)^{\mu}+\ell_{\alpha}^{2}\left(S_{\alpha} \wedge p_{\beta}\right)^{\mu}\right\}\right],
\end{aligned}
$$

where we have used the gauge freedom to add a term proportional to $k^{\mu}$, so that, on shell, the double copy satisfies the Ward identity in both indices.

We can use the double copy to obtain radiation amplitudes in various channels, by decomposing the product of polarizations in Eq. (35) into irreducible representations of the massless little group $S O(d-2)$ as

$$
\epsilon_{\mu} \tilde{\epsilon}_{\nu}=\epsilon_{\mu \nu}+a_{\mu \nu}+\frac{\epsilon \cdot \tilde{\epsilon}}{d-2} \pi_{\mu \nu}
$$

where $\epsilon_{\mu \nu} \equiv \frac{1}{2}\left(\epsilon_{\mu} \tilde{\epsilon}_{\nu}+\epsilon_{\nu} \tilde{\epsilon}_{\mu}\right)-\frac{\epsilon \cdot \tilde{\epsilon}}{d-2} \pi_{\mu \nu}, a_{\mu \nu} \equiv \frac{1}{2}\left(\epsilon_{\mu} \tilde{\epsilon}_{\nu}-\epsilon_{\nu} \tilde{\epsilon}_{\mu}\right)$, and $\frac{\epsilon \cdot \tilde{\epsilon}}{d-2} \pi_{\mu \nu}$ are the symmetric-traceless, the antisymmetric, and the trace parts, respectively. Here $\pi_{\mu \nu} \equiv \eta_{\mu \nu}-\frac{\left(k_{\mu} q_{\nu}+k_{\nu} q_{\mu}\right)}{k . q}$ is the projector onto the $(d-2)$-dimensional space spanned by the polarization vectors orthogonal to both the external momentum $k$ and an arbitrary reference vector $q$, satisfying $q^{2}=0$.

In the nonspinning case, it was shown, by an explicit computation, that the double copy produces nonvanishing radiation amplitudes in the dilaton and graviton channels of a gravitational theory whose action is $S=S_{g}+S_{p p}$, with

$$
S_{g}=-2 m_{\mathrm{Pl}}^{d-2} \int d^{d} x \sqrt{g}\left[R-(d-2) g^{\mu \nu} \partial_{\mu} \phi \partial_{\nu} \phi\right],
$$

and

$$
S_{p p}=-\sum_{\alpha} m_{\alpha} \int d \tau_{\alpha} e^{\phi}
$$

In $d=4$, for example, this means that the radiation fields at null infinity calculated in this dilaton gravity theory can be reproduced with the double copy, by writing

$$
\begin{gathered}
h_{ \pm}(t, \vec{n})=\frac{4 G_{N}}{r} \int \frac{d \omega}{2 \pi} e^{-i \omega t} \epsilon_{ \pm}^{* \mu \nu}(k) \mathcal{A}_{\mu \nu}(k) \\
\phi(t, \vec{n})=\frac{G_{N}}{r} \int \frac{d \omega}{2 \pi} e^{-i \omega t} \eta^{\mu \nu} \mathcal{A}_{\mu \nu}(k) .
\end{gathered}
$$

Here $\vec{n}=\vec{k} /|\vec{k}|$, and $\omega=k^{0}$ is the frequency of radiation (in $d=4, G_{N}=1 / 32 \pi m_{\mathrm{Pl}}^{2}$ ).

As discussed in the Introduction, we expect the double copy amplitude to also have a nonvanishing antisymmetric component. This corresponds to the Kalb-Ramond axion $B_{\mu \nu}(x)$ in the gravitational theory. Hence, our purported gravitational theory has the field content $\left(h_{\mu \nu}, B_{\mu \nu}, \phi\right)$. We now write down the radiation amplitudes in this theory, as predicted by the double copy.

The axion amplitude is defined to be

$$
\hat{\mathcal{A}}_{B}(k) \equiv a_{\mu \nu}^{*}(k) \mathcal{A}^{\mu \nu}(k)
$$

2-form gauge invariance implies that the polarization tensor is defined up to gauge transformations $a_{\mu \nu}(k) \rightarrow a_{\mu \nu}(k)+$ $k_{\mu} \zeta_{\nu}(k)-k_{\nu} \zeta_{\mu}(k)$ for an arbitrary gauge parameter $\zeta_{\nu}(k)$. Hence, the axion amplitude is predicted to be 


$$
\begin{aligned}
\left.\hat{\mathcal{A}}_{B}(k)\right|_{\mathcal{O}\left(\eta^{3}, S^{1}\right)}= & -\frac{i a_{\mu \nu}^{*}(k)}{16 m_{\mathrm{Pl}}^{3(d-2) / 2}} \sum_{\substack{\alpha, \beta \\
\alpha \neq \beta}} \int d \mu_{\alpha \beta}(k)\left[\left(\frac { \ell _ { \alpha } ^ { 2 } ( p _ { \alpha } \cdot p _ { \beta } ) } { k \cdot p _ { \alpha } } \left(p _ { \alpha } ^ { \nu } \left\{k \cdot \ell_{\beta}\left(\frac{p_{\alpha} \cdot p_{\beta}}{k \cdot p_{\alpha}}\left(S_{\alpha} \wedge k\right)^{\mu}-\left(S_{\alpha} \wedge p_{\beta}\right)^{\mu}\right)\right.\right.\right.\right. \\
& \left.\left.-\left(k \cdot p_{\beta}\right)\left(S_{\alpha} \wedge k\right)^{\mu}-\left(k \cdot p_{\beta}\right)\left(S_{\alpha} \wedge \ell_{\alpha}\right)^{\mu}+\frac{1}{2}\left(\ell_{\beta}^{\mu}-\ell_{\alpha}^{\mu}\right)\left(k \wedge p_{\beta}\right)_{\alpha}-p_{\beta}^{\mu}\left(\ell_{\alpha} \wedge \ell_{\beta}\right)_{\alpha}\right\}\right) \\
& \left.-\frac{1}{2}\left(p_{\alpha} \cdot p_{\beta}\right)\left(\ell_{\beta}-\ell_{\alpha}\right)^{\nu}\left(S_{\alpha} \wedge k\right)^{\mu}\right)+\ell_{\alpha}^{2}\left(( p _ { \alpha } \cdot p _ { \beta } ) \left(p_{\alpha}^{\nu}\left(S_{\beta} \wedge \ell_{\beta}\right)^{\mu}+p_{\beta}^{\nu}\left(S_{\alpha} \wedge k\right)^{\mu}\right.\right. \\
& \left.\left.-p_{\beta}^{\mu}\left(\ell_{\beta}-\ell_{\alpha}\right)^{\nu}\left(\ell_{\alpha} \wedge \ell_{\beta}\right)_{\alpha}\right)-p_{\beta}^{\nu} p_{\alpha}^{\mu}\left[\left(\ell_{\beta} \wedge p_{\alpha}\right)_{\beta}-\left(\ell_{\beta} \wedge p_{\beta}\right)_{\alpha}\right]\right) \\
& +\left(p_{\alpha}^{\nu}\left(k \cdot p_{\beta}\right)-p_{\beta}^{\nu}\left(k \cdot p_{\alpha}\right)+\frac{1}{2}\left(\ell_{\beta}^{\nu}-\ell_{\alpha}^{\nu}\right)\left(p_{\alpha} \cdot p_{\beta}\right)\right)\left(2\left(k \cdot p_{\alpha}\right)\left(S_{\beta} \wedge \ell_{\beta}\right)^{\mu}\right. \\
& \left.\left.+\ell_{\alpha}^{2}\left(S_{\alpha} \wedge p_{\beta}\right)^{\mu}\right)+\ell_{\beta}^{\mu}\left(\left(k \cdot p_{\beta}\right) p_{\alpha}^{\nu}-\left(k \cdot p_{\alpha}\right) p_{\beta}^{\nu}\right)\left[\left(\ell_{\alpha} \wedge p_{\beta}\right)_{\alpha}+\left(\ell_{\beta} \wedge p_{\alpha}\right)_{\beta}\right]-\{\mu \leftrightarrow \nu\}\right] .
\end{aligned}
$$

Similarly, the graviton amplitude is given by

$$
\hat{\mathcal{A}}_{g}(k) \equiv \epsilon_{\mu \nu}^{*} \mathcal{A}^{\mu \nu}(k),
$$

where the polarization tensor $\epsilon_{\mu \nu}(k)$ is defined up to gauge transformations $\epsilon_{\mu \nu}(k) \rightarrow \epsilon_{\mu \nu}(k)+k_{\mu} \zeta_{\nu}(k)+k_{\nu} \zeta_{\mu}(k)$ for an arbitrary gauge parameter $\zeta_{\nu}(k)$. The explicit prediction is

$$
\begin{aligned}
\left.\hat{\mathcal{A}}_{g}(k)\right|_{\mathcal{O}\left(\eta^{3}, S^{1}\right)}= & \frac{i \epsilon_{\mu \nu}^{*}}{8 m_{\mathrm{Pl}}^{3(d-2) / 2}} \sum_{\substack{\alpha \beta \beta \\
\alpha \neq \beta}} \int d \mu_{\alpha \beta}(k)\left[\frac { ( p _ { \alpha } \cdot p _ { \beta } ) ( k \cdot \ell _ { \beta } ) \ell _ { \alpha } ^ { 2 } } { 2 ( k \cdot p _ { \alpha } ) ^ { 2 } } \left\{\left(\left(\ell_{\beta} \wedge p_{\alpha}\right)_{\beta}-\left(\ell_{\beta} \wedge p_{\beta}\right)_{\alpha}\right) p_{\alpha}^{\mu} p_{\alpha}^{\nu}\right.\right. \\
& \left.-\left(p_{\alpha} \cdot p_{\beta}\right)\left(S_{\alpha} \wedge k\right)^{\mu} p_{\alpha}^{\nu}\right\}+\frac{p_{\alpha} \cdot p_{\beta}}{2\left(k \cdot p_{\alpha}\right)}\left\{\frac{1}{2} \ell_{\alpha}^{2}\left(p_{\alpha} \cdot p_{\beta}\right)\left(S_{\alpha} \wedge k\right)^{\mu}\left(\ell_{\beta}-\ell_{\alpha}\right)^{\nu}+\ell_{\alpha}^{2}\left(k \cdot p_{\beta}\right)\left(S_{\alpha} \wedge \ell_{\alpha}\right)^{\mu} p_{\alpha}^{\nu}\right. \\
& +\ell_{\alpha}^{2}\left(k \cdot p_{\beta}\right)\left(S_{\alpha} \wedge k\right)^{\mu} p_{\alpha}^{\nu}+\ell_{\alpha}^{2}\left(k \cdot \ell_{\beta}\right)\left(S_{\alpha} \wedge p_{\beta}\right)^{\mu} p_{\alpha}^{\nu}+\ell_{\alpha}^{2}\left(\ell_{\alpha} \wedge \ell_{\beta}\right)_{\alpha} p_{\alpha}^{\mu} p_{\beta}^{\nu}+\ell_{\alpha}^{2}\left(\ell_{\alpha} \wedge \ell_{\beta}\right)_{\beta} p_{\alpha}^{\mu} p_{\alpha}^{\nu} \\
& \left.-\left(\ell_{\alpha} \cdot \ell_{\beta}\right)\left(\ell_{\alpha} \wedge p_{\beta}\right)_{\alpha} k^{\mu} p_{\alpha}^{\nu}-\ell_{\alpha}^{2}\left(k \wedge p_{\beta}\right)_{\alpha} \ell_{\beta}^{\mu} p_{\alpha}^{\nu}+\ell_{\alpha}^{2}\left\{\left(\ell_{\beta} \wedge p_{\beta}\right)_{\alpha}-\left(\ell_{\beta} \wedge p_{\alpha}\right)_{\beta}\right\}\left(2 \ell_{\beta}-\frac{1}{2} k\right)^{\mu} p_{\alpha}^{\nu}\right\} \\
& +\frac{\left(k \cdot p_{\beta}\right) \ell_{\alpha}^{2}}{2\left(k \cdot p_{\alpha}\right)}\left\{\left(\ell_{\beta} \wedge p_{\beta}\right)_{\alpha}-\left(\ell_{\beta} \wedge p_{\alpha}\right)_{\beta}\right\} p_{\alpha}^{\mu} p_{\alpha}^{\nu}+\frac{1}{2}\left(p_{\alpha} \cdot p_{\beta}\right)\left\{-\ell_{\alpha}^{2}\left(S_{\alpha} \wedge k\right)^{\mu} p_{\beta}^{\nu}-\ell_{\alpha}^{2}\left(S_{\beta} \wedge \ell_{\beta}\right)^{\mu} p_{\alpha}^{\nu}\right. \\
& \left.+\left(\left(k \cdot p_{\beta}\right)\left(S_{\alpha} \wedge \ell_{\alpha}\right)^{\mu}-\frac{1}{2} \ell_{\alpha}^{2}\left(S_{\alpha} \wedge p_{\beta}\right)^{\mu}+\left(\ell_{\alpha} \wedge \ell_{\beta}\right)_{\alpha} p_{\beta}^{\mu}+\frac{1}{2}\left(\ell_{\alpha} \wedge p_{\beta}\right)_{\alpha}\left(\ell_{\alpha}-\ell_{\beta}\right)^{\mu}\right)\left(\ell_{\beta}-\ell_{\alpha}\right)^{\nu}\right\} \\
& -\frac{\ell_{\alpha}^{2}}{2}\left\{\left(\ell_{\beta} \wedge p_{\beta}\right)_{\alpha}-\left(\ell_{\beta} \wedge p_{\alpha}\right)_{\beta}\right\} p_{\alpha}^{\mu} p_{\beta}^{\nu}+\left\{\left(k \cdot p_{\beta}\right)\left(S_{\alpha} \wedge \ell_{\alpha}\right)^{\mu}-\frac{1}{2}\left(S_{\alpha} \wedge p_{\beta}\right)^{\mu}\right. \\
& \left.\left.+\left(\ell_{\alpha} \wedge \ell_{\beta}\right)_{\alpha} p_{\beta}^{\mu}+\left(\ell_{\alpha} \wedge p_{\beta}\right)_{\alpha}\left(\ell_{\alpha}-\frac{1}{2} k\right)^{\mu}\right\}\left(\left(k \cdot p_{\beta}\right) p_{\alpha}^{\nu}-\left(k \cdot p_{\alpha}\right) p_{\beta}^{\nu}\right)+(\mu \leftrightarrow \nu)\right] .
\end{aligned}
$$

Finally, the dilaton amplitude is defined to be

$$
\hat{\mathcal{A}}_{s}(k) \equiv \frac{1}{(d-2)^{1 / 2}} \eta_{\mu \nu} \mathcal{A}^{\mu \nu}(k) .
$$

This predicts the total dilaton amplitude as

$$
\begin{aligned}
\left.\hat{\mathcal{A}}_{s}(k)\right|_{\mathcal{O}\left(\eta^{3}, S^{1}\right)}= & -\frac{i}{8 m_{\mathrm{Pl}}^{3(d-2) / 2}(d-2)^{1 / 2}} \sum_{\substack{\alpha, \beta \\
\alpha \neq \beta}} \int d \mu_{\alpha \beta}(k) p_{\alpha}^{2}\left[\frac{\left(p_{\alpha} \cdot p_{\beta}\right)\left(k \cdot \ell_{\beta}\right) \ell_{\alpha}^{2}}{\left(k \cdot p_{\alpha}\right)^{2}}\left\{\left(\ell_{\beta} \wedge p_{\alpha}\right)_{\beta}-\left(\ell_{\beta} \wedge p_{\beta}\right)_{\alpha}\right\}\right. \\
& \left.+\frac{\ell_{\alpha}^{2}}{k \cdot p_{\alpha}}\left\{\left(p_{\alpha} \cdot p_{\beta}\right)\left(k \wedge \ell_{\beta}\right)_{\beta}+\left(k \cdot p_{\beta}\right)\left(\ell_{\beta} \wedge p_{\beta}\right)_{\alpha}-\left(k \cdot p_{\beta}\right)\left(\ell_{\beta} \wedge p_{\alpha}\right)_{\beta}\right\}+2\left(k \cdot p_{\beta}\right)\left(\ell_{\alpha} \wedge \ell_{\beta}\right)_{\beta}\right] .
\end{aligned}
$$




\section{THE GRAVITATIONAL THEORY}

In this section, we calculate the amplitudes for dilaton, graviton, and axion radiation emitted by a set of spinning sources coupled to gravity. We note that the color-kinematic substitution only contributes to additional powers of momenta in the numerators so it can only improve the analyticity of the amplitude. Hence, we expect the resulting gravitational theory to be local. The most general action in the bulk, up to two derivatives, with field content $\left(h_{\mu \nu}, B_{\mu \nu}, \phi\right)$ that preserves the symmetries, namely, diffeomorphism invariance and 2-form gauge invariance, is

$$
\begin{aligned}
S_{g}= & -2 m_{\mathrm{Pl}}^{d-2} \int d^{d} x \sqrt{g}\left[R-(d-2) g^{\mu \nu} \partial_{\mu} \phi \partial_{\nu} \phi\right. \\
& \left.+\frac{1}{12} f(\phi) H_{\mu \nu \sigma} H^{\mu \nu \sigma}\right]
\end{aligned}
$$

where $H_{\mu \nu \sigma}=(d B)_{\mu \nu \sigma}$ is the field strength corresponding to the 2 -form field and $f(\phi)=1+f^{\prime}(0) \phi+\cdots$.

Next, we move to the point particle action. We calculate Yang-Mills radiation to linear order in spin, whereas, on the gravitational side, it is easy to see that the leading order interaction of gravitons with the spin is second order in spin. However, the double copy predicts a nonvanishing axion amplitude linear in spin. This suggests that the corresponding gravity theory has a linear interaction of the spin with the 2-form field. In [20], we wrote this unique leading order interaction term as

$$
S_{H S}=\int d x^{\mu} \tilde{\kappa}(\phi) H_{\mu \nu \sigma} \tilde{S}^{\nu \sigma},
$$

for an arbitrary function $\tilde{\kappa}(\phi)=\tilde{\kappa}+\tilde{\kappa}^{\prime} \phi+\cdots$. We used $\tilde{S}^{\mu \nu}=S^{I J} \tilde{e}_{I}^{\mu} \tilde{e}_{J}^{\nu}$, with $\tilde{e}_{I}^{\mu}$ being the vielbeins defined with respect to the string frame metric, i.e., $\eta_{I J} \tilde{e}_{\mu}^{I} \tilde{e}_{\nu}^{J}=\tilde{g}_{\mu \nu}=g_{\mu \nu} e^{2 \phi}$. The double copied field does not have any free parameters. This means that consistency with the double copy should fix all the unknown parameters on the gravitational side. Indeed, comparing the axion radiation amplitude in this theory to the double copy prediction, these parameters were fixed to be [20]

$$
f^{\prime}(0)=-4, \quad \tilde{\kappa}^{\prime}=0, \quad \tilde{\kappa}=\frac{1}{4} .
$$

The bulk action is then given by

$$
\begin{aligned}
S_{g}= & -2 m_{\mathrm{Pl}}^{d-2} \int d^{d} x \sqrt{g}\left[R-(d-2) g^{\mu \nu} \partial_{\mu} \phi \partial_{\nu} \phi\right. \\
& \left.+\frac{e^{-4 \phi}}{12} H_{\mu \nu \rho} H^{\mu \nu \rho}\right] .
\end{aligned}
$$

In this paper, we define the spin via $S^{\mu \nu}=S^{I J} e_{I}^{\mu} e_{J}^{\nu}$, where the vielbeins $e_{\mu}^{I}$ are defined with respect to the ordinary metric, $\eta_{I J} e_{\mu}^{I} e_{\nu}^{J}=g_{\mu \nu}$. Then, the complete worldline action for a single particle is

$$
\begin{aligned}
S_{p p}= & \int d s\left[-\dot{x}^{\mu} e_{\mu}^{I} p_{I} e^{\phi}+\frac{1}{2} S^{I J} \Omega_{I J}+\frac{1}{2} e\left(p^{I} p_{I}-m^{2}\right) e^{\phi}\right. \\
& \left.+e \lambda_{I} S^{I J} p_{J}+\frac{1}{4} S^{\mu \nu} \dot{x}^{\sigma} H_{\mu \nu \sigma} e^{-2 \phi}\right] .
\end{aligned}
$$

Here, the angular velocity is defined with a covariant derivative, $\Omega_{I J}=g_{\mu \nu} e_{I}^{\mu} \frac{D}{D s} e_{J}^{\nu} \equiv e_{I}^{\mu} \dot{x}_{\rho} \nabla^{\rho} e_{J}^{\nu}$. Note that though the unknown dilaton dependent functions in the action above have been written as exponentials, our computation only really fixes these functions to linear order in the dilaton. We expect the complete bulk action to be given by Eq. (53) as it describes the BCJ double copy of pure Yang-Mills [32]. It also arises as the leading low energy effective theory of the common sector of oriented string theories [33,34].

In the next subsection, we find the equations of motion for the system of fields and particles. We work with the ordinary metric and, later, fix the worldline parameter $s$ to be the proper time per unit mass, $s=\tau$. This ensures that we get the nonspinning action and equations of motion in [14] when the spin is set to zero and particle masses are restored. To get the relevant results in [20], we only need to switch back to the conformally rescaled metric $\tilde{g}_{\mu \nu}=$ $g_{\mu \nu} e^{2 \phi}$ and reparametrize the worldline coordinate to be the conformal proper time per unit mass, $s=\tilde{\tau} e^{-\phi}$. Of course, worldline reparametrization invariance ensures the invariance of the total amplitude. In Sec. IV B, we work out the leading order fields and the changes they induce in the momenta, color, and spin of the particles. We use these field values and particle deflections to calculate the leading order axion, graviton, and dilaton radiation in Secs. IV C, IV D, and IV E, respectively.

\section{A. Equations of motion and solutions}

The equation of motion for the dilaton is

$\nabla^{\mu} \nabla_{\mu} \phi-\frac{e^{-4 \phi}}{6(d-2)} H_{\mu \nu \rho} H^{\mu \nu \rho}=-\frac{1}{4 m_{\mathrm{Pl}}^{d-2}(d-2)} J$,

where we have defined the source term on the rhs to be

$J \equiv \sum_{\alpha} \int d s\left(\dot{x}_{\alpha}^{\mu} p_{\alpha \mu} e^{\phi}+S_{\alpha}^{\mu \nu} \dot{x}_{\alpha}^{\sigma} H_{\mu \nu \sigma} e^{-2 \phi}\right) \frac{\delta^{d}\left(x-x_{\alpha}\right)}{\sqrt{g}}$.

We also derive the equation of motion of the axion to be

$$
\nabla_{\lambda}\left(e^{-4 \phi} H^{\mu \nu \lambda}\right)=\frac{1}{m_{\mathrm{Pl}}^{d-2}} J^{\mu \nu},
$$

whose source term is defined to be 


$$
\begin{aligned}
J^{\mu \nu} \equiv & \sum_{\alpha} \frac{1}{4} \int d s\left(S_{\alpha}^{\lambda \mu} \dot{x}_{\alpha}^{\nu}+S_{\alpha}^{\nu \lambda} \dot{x}_{\alpha}^{\mu}+S_{\alpha}^{\mu \nu} \dot{x}_{\alpha}^{\lambda}\right) \nabla_{\lambda} \\
& \times\left[e^{-2 \phi} \frac{\delta^{d}\left(x-x_{\alpha}\right)}{\sqrt{g}}\right]
\end{aligned}
$$

We now find the equations of motion for the particles using the same method as in [20]. First, we write down the energy-momentum tensor for the point particles as

$$
\begin{aligned}
T_{p p}^{\mu \nu}= & -\frac{2}{\sqrt{g}} \frac{\delta}{\delta g_{\mu \nu}(x)} S_{p p}=\sum_{\alpha} \int d x_{\alpha}^{(\mu} p_{\alpha}^{\nu)} \frac{\delta^{d}\left(x-x_{\alpha}\right)}{\sqrt{g}} e^{\phi} \\
& +\int d x_{\alpha}^{(\mu} S_{\alpha}^{\nu) \alpha} \nabla_{\alpha}\left[\frac{\delta^{d}\left(x-x_{\alpha}\right)}{\sqrt{g}}\right] \\
& -\frac{1}{2} \int d x_{\alpha}^{\sigma} H_{\rho \lambda \sigma} g^{\lambda(\mu} S_{\alpha}^{\nu) \rho} e^{-2 \phi} \frac{\delta^{d}\left(x-x_{\alpha}\right)}{\sqrt{g}}
\end{aligned}
$$

where the first line is the result for dilaton gravity, and the second line includes the contribution of the axion. We can also write down the contributions to the energy-momentum tensor from the axion and the dilaton, respectively, as
$T_{B}^{\mu \nu}=-m_{\mathrm{Pl}}^{d-2} e^{-4 \phi} g_{\rho \sigma} g_{\tau \lambda} H^{\mu \rho \tau} H^{\nu \sigma \lambda}+\frac{m_{\mathrm{Pl}}^{d-2}}{6} e^{-4 \phi} H^{2} g^{\mu \nu}$

and

$T_{\phi}^{\mu \nu}=(d-2) m_{\mathrm{Pl}}^{d-2}\left(4 \partial^{\mu} \phi \partial^{\nu} \phi-2 g^{\mu \nu} g^{\rho \sigma} \partial_{\rho} \phi \partial_{\sigma} \phi\right)$.

Using the equations of motion for the axion and the dilaton, we see that

$$
\nabla_{\mu} T_{B}^{\mu \nu}=J_{\sigma \rho} H^{\nu \sigma \rho}
$$

and

$$
\nabla_{\mu} T_{\phi}^{\mu \nu}=J \partial^{\nu} \phi
$$

Now, we obtain the particle equations of motion by integrating $\nabla_{\mu}\left(T_{p p}^{\mu \nu}+T_{B}^{\mu \nu}+T_{\phi}^{\mu \nu}\right)=0$ with an arbitrary vector $X^{\mu}(x)$, to get

$$
\begin{aligned}
\int d^{d} x \sqrt{g} X_{\nu} \nabla_{\mu}\left(T_{p p}^{\mu \nu}+T_{B}^{\mu \nu}+T_{\phi}^{\mu \nu}\right)= & \int d^{d} x \sqrt{g} X_{\nu}\left(\nabla_{\mu} T_{p p}^{\mu \nu}-J_{\sigma \rho} H^{\nu \sigma \rho}-J \partial^{\nu} \phi\right) \\
= & \sum_{\alpha} \int d s\left[\nabla_{\mu} X_{\nu}\left(-\frac{1}{2} \dot{x} \cdot \nabla\left(S_{\alpha}^{\nu \mu}\right)+\frac{1}{2} H_{\lambda \sigma \rho} g^{\lambda[\mu} S_{\alpha}^{\nu] \rho} \dot{x}_{\alpha}^{\sigma} e^{-2 \phi}+\dot{x}_{\alpha}^{[\mu} p_{\alpha}^{\nu]} e^{\phi}\right)\right. \\
& +X_{\nu}\left(\dot{x}_{\alpha} \cdot \nabla\left(p_{\alpha}^{\nu} e^{\phi}\right)-\frac{1}{2} R_{\mu \lambda \sigma}^{\nu} \dot{x}_{\alpha}^{\mu} S_{\alpha}^{\sigma \lambda}-\partial^{\nu} \phi\left(\dot{x}_{\alpha}^{\mu} p_{\alpha \mu} e^{\phi}+S_{\alpha}^{\mu \rho} \dot{x}_{\alpha}^{\sigma} H_{\mu \rho \sigma} e^{-2 \phi}\right)\right. \\
& \left.\left.-\frac{1}{4} \dot{x}_{\alpha} \cdot \nabla\left(H_{\lambda \sigma \rho} g^{\nu \lambda} S_{\alpha}^{\sigma \rho} e^{-2 \phi}\right)\right)\right] .
\end{aligned}
$$

Since the vector $X^{\mu}(x)$ is arbitrary, we can equate the coefficients of $X^{\mu}(x)$ and $\nabla_{\mu} X_{\nu}$ to zero, to get the exact equations for spin and momentum,

$$
\begin{gathered}
\frac{d S_{\alpha}^{\mu \nu}}{d s}=p_{\alpha}^{\mu} \dot{x}_{\alpha}^{\nu} e^{\phi}-p_{\alpha}^{\nu} \dot{x}_{\alpha}^{\mu} e^{\phi}-\Gamma_{\sigma \rho}^{\mu} S_{\alpha}^{\rho \nu} \dot{x}_{\alpha}^{\sigma}-\Gamma_{\sigma \rho}^{\nu} S_{\alpha}^{\mu \rho} \dot{x}_{\alpha}^{\sigma}-\frac{1}{2} H_{\lambda \sigma \rho} g^{\lambda \mu} S_{\alpha}^{\nu \rho} \dot{x}_{\alpha}^{\sigma} e^{-2 \phi}+\frac{1}{2} H_{\lambda \sigma \rho} g^{\lambda \nu} S_{\alpha}^{\mu \rho} \dot{x}_{\alpha}^{\sigma} e^{-2 \phi}, \\
\frac{d p_{\alpha}^{\mu}}{d s}=p_{\alpha \nu} \dot{x}_{\alpha}^{\nu} \partial^{\mu} \phi-p_{\alpha}^{\mu} \dot{x}_{\alpha}^{\sigma} \partial_{\sigma} \phi-\Gamma_{\sigma \rho}^{\mu} \dot{x}_{\alpha}^{\sigma} p_{\alpha}^{\rho}+\frac{1}{2} R_{\nu \lambda \sigma}^{\mu} \dot{x}_{\alpha}^{\nu} S_{\alpha}^{\sigma \lambda} e^{-\phi}+\frac{1}{4} \dot{x}_{\alpha} \cdot \nabla\left(g^{\mu \lambda} H_{\lambda \sigma \rho} S_{\alpha}^{\sigma \rho} e^{-2 \phi}\right) e^{-\phi}-\frac{1}{2} S_{\alpha}^{\lambda \rho} \dot{x}_{\alpha}^{\sigma} H_{\lambda \rho \sigma} e^{-3 \phi} \partial^{\mu} \phi .
\end{gathered}
$$

We rewrite the dilaton equation of motion (55) as

$$
\square \phi(x) \equiv-\frac{1}{4 m_{\mathrm{Pl}}^{d-2}(d-2)} \tilde{J}(x)
$$

where $\square \equiv \eta^{\mu \nu} \partial_{\mu} \partial_{\nu}$, and we have defined $\tilde{J}(x)$ to include axion and graviton contributions from the lhs of Eq. (55). With the dilaton propagator

$$
\langle\phi(k) \phi(-k)\rangle=\frac{i}{4 m_{\mathrm{Pl}}^{d-2}(d-2) k^{2}},
$$


we can formally write the solution as

$$
\langle\phi\rangle(x)=-\frac{1}{2 m_{\mathrm{Pl}}^{(d-2) / 2}(d-2)^{1 / 2}} \int_{k} \frac{e^{-i k \cdot x}}{k^{2}} \mathcal{A}_{s}(k),
$$

thereby defining a canonically normalized dilaton radiation amplitude $\quad \mathcal{A}_{s}(k)=-\frac{1}{2 m_{\mathrm{Pl}}^{(d-2) / 2}(d-2)^{1 / 2}} \tilde{J}(k) \quad$ for $\quad$ on-shell momentum $k^{2}=0$.

Choosing the gauge $\partial_{\mu} B^{\mu \nu}=0$, we rewrite the equation of motion for the axion, Eq. (57) as

$$
\square B^{\mu \nu}(x) \equiv \frac{1}{m_{\mathrm{Pl}}^{d-2}} \tilde{J}^{\mu \nu}(x) .
$$

The gauge condition ensures that the classical axion current $\tilde{J}^{\mu \nu}(x)$, so defined, satisfies the conservation equation $\partial_{\mu} \tilde{J}^{\mu \nu}(x)=0$. In this gauge, the propagator for the axion field is

$$
\left\langle B_{\mu \nu}(k) B_{\rho \sigma}(-k)\right\rangle=\frac{i}{2 m_{\mathrm{Pl}}^{d-2} k^{2}}\left[\eta_{\mu \rho} \eta_{\nu \sigma}-\eta_{\mu \sigma} \eta_{\nu \rho}\right] .
$$

Then, the formal solution to Eq. (70) is

$$
\left\langle B^{\mu \nu}\right\rangle(x)=-\frac{1}{m_{\mathrm{Pl}}^{d-2}} \int_{k} \frac{e^{-i k \cdot x}}{k^{2}} \tilde{J}^{\mu \nu}(k),
$$

which defines an axion radiation amplitude $\mathcal{A}_{B}=$ $\frac{1}{m_{\mathrm{Pl}}^{(d-2) / 2}} a_{\mu \nu}^{*} \tilde{J}^{\mu \nu}(k)$ for on-shell momentum $k^{2}=0$.

Finally, we get to the gravitational field. We expand the metric perturbatively about flat space $g_{\mu \nu}=\eta_{\mu \nu}+h_{\mu \nu}$. We choose the de-Donder gauge $\partial_{\mu} h^{\mu \nu}=\frac{1}{2} \partial_{\mu} h_{\lambda}^{\lambda}$, in which we have the propagator

$$
\begin{aligned}
& \left\langle h_{\mu \nu}(k) h_{\rho \sigma}(-k)\right\rangle \\
& \quad=\frac{i}{2 m_{\mathrm{Pl}}^{d-2} k^{2}}\left[\eta_{\mu \rho} \eta_{\nu \sigma}+\eta_{\mu \sigma} \eta_{\nu \rho}-\frac{2}{d-2} \eta_{\mu \nu} \eta_{\rho \sigma}\right],
\end{aligned}
$$

and the solution for $h_{\mu \nu}(x)$ can be written formally as

$$
\left\langle h_{\mu \nu}\right\rangle(x)=\frac{1}{2 m_{\mathrm{Pl}}^{d-2}} \int \frac{e^{-i k \cdot x}}{k^{2}}\left\{\tilde{T}_{\mu \nu}(k)-\frac{1}{d-2} \eta_{\mu \nu} \tilde{T}_{\sigma}^{\sigma}\right\} .
$$

Here, $\tilde{T}_{\mu \nu}(k)$ is the energy-momentum pseudotensor that includes contributions from the sources as well as all the fields. It is (noncovariantly) conserved, $\partial_{\mu} \tilde{T}^{\mu \nu}=0$, and coordinate dependent, and hence it is nonunique. However, quantities such as energy-momentum or angular momentum can be defined as suitable integrals of $\tilde{T}^{\mu \nu}$ over spacetime. As in [11], we compute the background field gauge effective action [35], expressed as

$$
\Gamma(h, \phi, B)=-\frac{1}{2} \int d^{d} x \tilde{T}^{\mu \nu} h_{\mu \nu}
$$

so that the energy-momentum pseudotensor is related to the coefficient of the graviton one-point function. It is also directly related to the graviton amplitude by $\mathcal{A}_{g}=-\frac{1}{2 m_{\mathrm{Pl}}^{(d-2) / 2}} \epsilon_{\mu \nu}^{*} \tilde{T}^{\mu \nu}(k)$.

Observables at infinity are obtained in a manner similar to Eq. (20) by replacing the gluon source current and polarization vectors with the corresponding gravity ones. Hence, in the following, we solve for these sources $\tilde{T}^{\mu \nu}(k)$, $\tilde{J}^{\mu \nu}(k)$, and $\tilde{J}(k)$, in a perturbative expansion in powers of $\eta$, defined in Eq. (34), and to linear order in spin.

\section{B. Leading order results}

We now find the leading order solutions to the fields and the particle equations of motion. At leading order in perturbation, the axion field only gets a contribution from Fig. 2(a),

$$
\begin{aligned}
\left.\left\langle B^{\mu \nu}\right\rangle(x)\right|_{\mathcal{O}\left(\eta^{2}\right)} & \\
= & \frac{i}{4 m_{\mathrm{Pl}}^{d-2}} \sum_{\alpha} \int \frac{d^{d} \ell}{(2 \pi)^{d}} \frac{e^{-i \ell \cdot\left(x-x_{\alpha}\right)}}{\ell^{2}} \\
& \times\left[\left(S_{\alpha} \wedge \ell\right)^{\mu} p_{\alpha}^{\nu}-\left(S_{\alpha} \wedge \ell\right)^{\nu} p_{\alpha}^{\mu}-S_{\alpha}^{\mu \nu}\left(\ell \cdot p_{\alpha}\right)\right],
\end{aligned}
$$
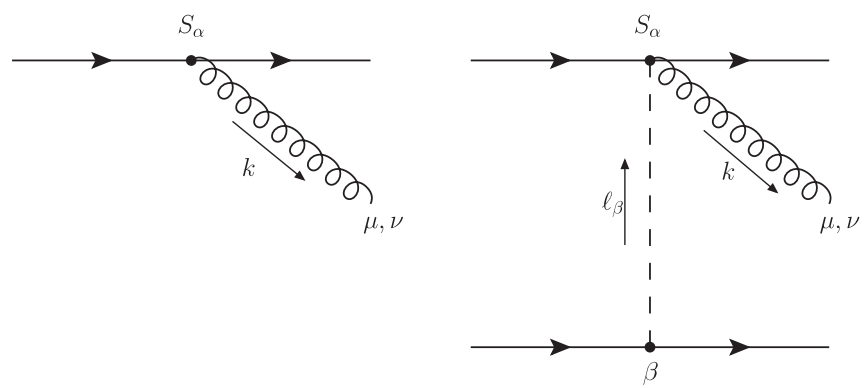

(a)

(b)

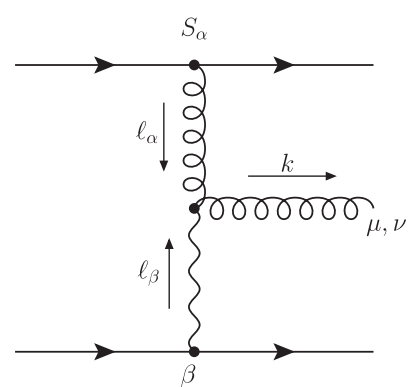

(c)

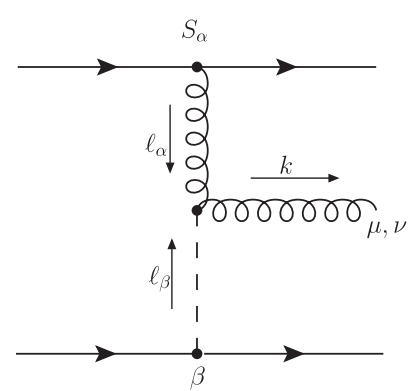

(d)
FIG. 2. Feynman diagrams for the perturbative expansion of the axion source current $\widetilde{J}^{\mu \nu}(k)$ up to $\mathcal{O}\left(\eta^{2}\right)$ and to linear order in spin. Here, wavy lines, curvy lines and dashed lines respectively represent gravitons, axions and dilatons. Diagrams (a),(b) represent contributions to axion radiation coming directly o the worldline. Diagrams (c),(d) represent contributions to axion radiation via 3 -point vertices involving the dilaton and graviton. 
for general time-dependent dynamical variables $p_{\alpha}^{\mu}$ and $S_{\alpha}^{\mu \nu}$ that satisfy the equations of motion.

The leading order metric perturbation contains a spinning and a nonspinning contribution sourced by Figs. 2(a) and 2(b), respectively. Their sum is given by

$$
\left.\left\langle h^{\mu \nu}\right\rangle(x)\right|_{\mathcal{O}\left(\eta^{2}\right)}=\frac{1}{2 m_{\mathrm{Pl}}^{d-2}} \sum_{\alpha} \int \frac{d^{d} \ell}{(2 \pi)^{d}} \frac{e^{-i \ell \cdot\left(x-x_{\alpha}\right)}}{\ell^{2}}\left[p_{\alpha}^{\mu} p_{\alpha}^{\nu}-\frac{m_{\alpha}^{2}}{d-2} \eta^{\mu \nu}-\frac{i}{2}\left\{p_{\alpha}^{\mu}\left(S_{\alpha} \wedge \ell\right)^{\nu}+p_{\alpha}^{\nu}\left(S_{\alpha} \wedge \ell\right)^{\mu}\right\}\right] .
$$

The leading order dilaton solution has no spin-dependent contribution, so it is the same as in the nonspinning case [14],

$$
\left.\langle\phi\rangle(x)\right|_{\mathcal{O}\left(\eta^{2}\right)}=\frac{1}{4 m_{\mathrm{Pl}}^{d-2}(d-2)} \sum_{\alpha} m_{\alpha}^{2} \int \frac{d^{d} \ell}{(2 \pi)^{d}} \frac{e^{-i \ell \cdot\left(x-x_{\alpha}\right)}}{\ell^{2}} .
$$

Next, we calculate the corrections in spin and momentum that these leading order fields induce. First, let us write the equations of motion for the particle up to linear order in spin,

$$
\begin{gathered}
\left.\frac{d}{d s} S_{\alpha}^{\mu \nu}\right|_{\mathcal{O}\left(S^{0}\right)+\mathcal{O}\left(S^{1}\right)}=p_{\alpha}^{\mu} v_{\alpha}^{\nu} e^{\phi}-p_{\alpha}^{\nu} v_{\alpha}^{\mu} e^{\phi}-\Gamma_{\sigma \rho}^{\mu} S_{\alpha}^{\rho \nu} v_{\alpha}^{\sigma}-\Gamma_{\sigma \rho}^{\nu} S_{\alpha}^{\mu \rho} v_{\alpha}^{\sigma}, \\
\left.\frac{d}{d s} p_{\alpha}^{\mu}\right|_{\mathcal{O}\left(S^{0}\right)+\mathcal{O}\left(S^{1}\right)}=p_{\alpha \nu} v_{\alpha}^{\nu} \partial^{\mu} \phi-p_{\alpha}^{\mu} v_{\alpha}^{\sigma} \partial_{\sigma} \phi-\Gamma_{\sigma \rho}^{\mu} v_{\alpha}^{\sigma} p_{\alpha}^{\rho}+\frac{1}{2} R_{\nu \lambda \sigma}^{\mu} v_{\alpha}^{\nu} S_{\alpha}^{\sigma \lambda} e^{-\phi} .
\end{gathered}
$$

Inserting the leading order fields into the above equations gives the leading order changes in momenta and spin. First, for the momenta, we have

$$
\begin{aligned}
&\left.\frac{d}{d \tau_{\alpha}} p_{\alpha}^{\mu}\right|_{\mathcal{O}\left(\eta^{2}, S^{0}\right)}=\frac{i}{4 m_{\mathrm{Pl}}^{d-2}} \sum_{\beta \neq \alpha} \int d \tau_{\beta} \frac{d^{d} \ell}{(2 \pi)^{d}} \frac{e^{-i \ell \cdot x_{\alpha \beta}}}{\ell^{2}}\left[-\frac{m_{\beta}^{2}}{d-2}\left(\ell \cdot p_{\alpha}\right) p_{\alpha}^{\nu}+2\left(p_{\alpha} \cdot p_{\beta}\right)\left(\ell \cdot p_{\alpha}\right) p_{\beta}^{\nu}-\left(p_{\alpha} \cdot p_{\beta}\right)^{2} \ell^{\nu}\right], \\
&\left.\frac{d}{d \tau_{\alpha}} p_{\alpha}^{\mu}\right|_{\mathcal{O}\left(\eta^{2}, S^{1}\right)}= \frac{1}{4 m_{\mathrm{Pl}}^{d-2}} \sum_{\beta \neq \alpha} \int d \tau_{\beta} \frac{d^{d} \ell}{(2 \pi)^{d}} \frac{e^{-i \ell \cdot x_{\alpha \beta}}}{\ell^{2}}\left[\frac{m_{\beta}^{2}}{d-2}\left(\ell \cdot p_{\alpha}\right)\left(S_{\alpha} \wedge \ell\right)^{\mu}\right. \\
&\left.+\left[\left(\ell \wedge p_{\beta}\right)_{\alpha}-\left(\ell \wedge p_{\alpha}\right)_{\beta}\right]\left[\left(\ell \cdot p_{\alpha}\right) p_{\beta}^{\mu}-\left(p_{\alpha} \cdot p_{\beta}\right) \ell^{\mu}\right]+\left(\ell \cdot p_{\alpha}\right)\left(p_{\alpha} \cdot p_{\beta}\right)\left(S_{\beta} \wedge \ell\right)^{\mu}\right] .
\end{aligned}
$$

From this, we have the position equations of motion

$$
\begin{gathered}
v_{\alpha}^{\mu}=p_{\alpha}^{\mu}+\left.w_{\alpha}^{\mu}\right|_{\mathcal{O}\left(\eta^{2}, S^{1}\right)} \\
\left.w_{\alpha}^{\mu}\right|_{\mathcal{O}\left(\eta^{2}, S^{1}\right)} \equiv-\frac{i}{4 m_{\mathrm{Pl}}^{d-2}(d-2)} \sum_{\beta \neq \alpha} m_{\beta}^{2} \int d \tau_{\beta} \frac{d^{d} \ell}{(2 \pi)^{d}} \frac{e^{-i \ell \cdot x_{\alpha \beta}}}{\ell^{2}}\left(S_{\alpha} \wedge \ell\right)^{\mu} .
\end{gathered}
$$

Similarly, the spin equation of motion is given by

$$
\begin{aligned}
\left.\frac{d}{d \tau_{\alpha}} S_{\alpha}^{\mu \nu}\right|_{\mathcal{O}\left(\eta^{2}, S^{1}\right)}= & \left.p_{\alpha}^{\mu} w_{\alpha}^{\nu}\right|_{\mathcal{O}\left(\eta^{2}, S^{1}\right)}-\left.p_{\alpha}^{\nu} w_{\alpha}^{\mu}\right|_{\mathcal{O}\left(\eta^{2}, S^{1}\right)} \\
& +\frac{i}{4 m_{\mathrm{Pl}}^{d-2}} \sum_{\beta \neq \alpha} \int d \tau_{\beta} \frac{d^{d} \ell}{(2 \pi)^{d}} \frac{e^{-i \ell \cdot x_{\alpha \beta}}}{\ell^{2}}\left[\left(\ell \cdot p_{\alpha}\right)\left\{p_{\beta}^{\nu}\left(S_{\alpha} \wedge p_{\beta}\right)^{\mu}-p_{\beta}^{\mu}\left(S_{\alpha} \wedge p_{\beta}\right)^{\nu}\right\}\right. \\
& +\left(p_{\alpha} \cdot p_{\beta}\right)\left\{p_{\beta}^{\nu}\left(S_{\alpha} \wedge \ell\right)^{\mu}-p_{\beta}^{\mu}\left(S_{\alpha} \wedge \ell\right)^{\nu}+\ell^{\mu}\left(S_{\alpha} \wedge p_{\beta}\right)^{\nu}-\ell^{\nu}\left(S_{\alpha} \wedge p_{\beta}\right)^{\mu}\right\} \\
& \left.+\frac{m_{\beta}^{2}}{d-2}\left\{p_{\alpha}^{\mu}\left(S_{\alpha} \wedge \ell\right)^{\nu}-p_{\alpha}^{\nu}\left(S_{\alpha} \wedge \ell\right)^{\mu}-\ell^{\mu}\left(S_{\alpha} \wedge p_{\alpha}\right)^{\nu}+\ell^{\nu}\left(S_{\alpha} \wedge p_{\alpha}\right)^{\mu}-2\left(\ell \cdot p_{\alpha}\right) S_{\alpha}^{\mu \nu}\right\}\right] .
\end{aligned}
$$




\section{Axion radiation}

The leading order radiation has contributions from Figs. 2(a)-(d). The contribution from Fig. 2(a) is due to deflections in the position and spin of the particles, induced by the leading order fields. It is given by

$$
\begin{aligned}
\left.\tilde{J}^{\mu \nu}(k)\right|_{\text {Fig. } 2(\mathrm{a}) ; \mathcal{O}\left(\eta^{2}, S^{1}\right)}= & \frac{i}{4} \sum_{\alpha} \int d \tau_{\alpha} e^{i k \cdot x_{\alpha}} \frac{k_{\lambda}}{k \cdot v_{\alpha}}\left[-\frac{k \cdot \dot{v}_{\alpha}}{k \cdot v_{\alpha}} S_{\alpha}^{\lambda \mu} v_{\alpha}^{\nu}+S_{\alpha}^{\lambda \mu} \dot{v}_{\alpha}^{\nu}\right. \\
& \left.+\dot{S}_{\alpha}^{\lambda \mu} v_{\alpha}^{\nu}+\text { cyclic permutations }(\mu, \nu, \lambda)\right]\left.\right|_{\mathcal{O}\left(\eta^{2}, S^{1}\right)} .
\end{aligned}
$$

We can explicitly evaluate these expressions by substituting the leading order changes in particle spins and momenta, derived in Sec. IV B. The resulting expressions are given by Eqs. (A3) and (A4) in Appendix A 2.

The other contributions to the axion amplitude, at this order in perturbation, come from diagrams with no deflections in the trajectories of the particles. Figure 2(b), with an intermediate dilaton, corresponds to

$$
\left.\tilde{J}^{\mu \nu}(k)\right|_{\text {Fig. } 2(\mathrm{~b}) ; \mathcal{O}\left(\eta^{2}, S^{1}\right)}=-\frac{i}{16 m_{\mathrm{Pl}}^{d-2}(d-2)} \sum_{\substack{\alpha, \beta \\ \alpha \neq \beta}} m_{\beta}^{2} \int d \mu_{\alpha \beta}(k) \ell_{\alpha}^{2}\left[\left(k \cdot p_{\alpha}\right) S_{\alpha}^{\mu \nu}-2\left(S_{\alpha} \wedge k\right)^{\mu} p_{\alpha}^{\nu}-(\mu \leftrightarrow \nu)\right] .
$$

The two three-point vertex diagrams in Figs. 2(c) and 2(d) contribute

$$
\begin{aligned}
& \left.\tilde{J}^{\mu \nu}(k)\right|_{\text {Fig. } 2(\mathrm{c}) ; \mathcal{O}\left(\eta^{2}, S^{1}\right)}=-\frac{i}{4 m_{\mathrm{Pl}}^{d-2}(d-2)} \sum_{\substack{\alpha, \beta \\
\alpha \neq \beta}} m_{\beta}^{2} \int d \mu_{\alpha \beta}(k)\left[\left\{\left(k \cdot \ell_{\alpha}\right)\left(S_{\alpha} \wedge \ell_{\alpha}\right)^{\mu} p_{\alpha}^{\nu}\right.\right. \\
& \left.\left.-\left(k \cdot p_{\alpha}\right)\left(S_{\alpha} \wedge \ell_{\alpha}\right)^{\mu} \ell_{\alpha}^{\nu}+\left(k \wedge \ell_{\alpha}\right)_{\alpha} p_{\alpha}^{\mu} \ell_{\alpha}^{\nu}\right\}-(\mu \leftrightarrow \nu)\right], \\
& \left.\tilde{J}^{\mu \nu}(k)\right|_{\text {Fig. } 2(\mathrm{~d}) ; \mathcal{O}\left(\eta^{2}, S^{1}\right)}=-\frac{i}{8 m_{\mathrm{Pl}}^{d-2}} \sum_{\substack{\alpha, \beta \\
\alpha \neq \beta}} \int d \mu_{\alpha \beta}(k)\left[( p _ { \alpha } \cdot p _ { \beta } ) \left\{\left(k \cdot \ell_{\alpha}\right)\left(S_{\alpha} \wedge \ell_{\alpha}\right)^{\mu} p_{\beta}^{\nu}-\left(k \cdot p_{\beta}\right)\left(S_{\alpha} \wedge \ell_{\alpha}\right)^{\mu} \ell_{\alpha}^{\nu}\right.\right. \\
& \left.-\left(k \wedge \ell_{\alpha}\right)_{\alpha} l_{\alpha}^{\mu} p_{\beta}^{\nu}\right\}+\left(k \cdot p_{\beta}\right)^{2}\left(S_{\alpha} \wedge \ell_{\alpha}\right)^{\mu} p_{\alpha}^{\nu}-\left(k \cdot p_{\alpha}\right)\left(k \cdot p_{\beta}\right)\left(S_{\alpha} \wedge \ell_{\alpha}\right)^{\mu} p_{\beta}^{\nu} \\
& +\left(k \cdot p_{\beta}\right)\left(k \wedge \ell_{\alpha}\right)_{\alpha} p_{\alpha}^{\mu} p_{\beta}^{\nu}+\left(k \cdot p_{\beta}\right)\left(\ell_{\alpha} \wedge p_{\beta}\right)_{\alpha} \ell_{\alpha}^{\mu} p_{\alpha}^{\nu}-\left(k \cdot p_{\alpha}\right)\left(\ell_{\alpha} \wedge p_{\beta}\right)_{\alpha} \ell_{\alpha}^{\mu} p_{\beta}^{\nu} \\
& +\left(k \cdot \ell_{\alpha}\right)\left(\ell_{\alpha} \wedge p_{\beta}\right)_{\alpha} p_{\alpha}^{\mu} p_{\beta}^{\nu}-\frac{2 m_{\beta}^{2}}{d-2}\left\{\left(k \cdot \ell_{\alpha}\right)\left(S_{\alpha} \wedge \ell_{\alpha}\right)^{\mu} p_{\alpha}^{\nu}-\left(k \cdot p_{\alpha}\right)\left(S_{\alpha} \wedge \ell_{\alpha}\right)^{\mu} \ell_{\alpha}^{\nu}\right. \\
& \left.\left.+\left(k \wedge \ell_{\alpha}\right)_{\alpha} p_{\alpha}^{\mu} \ell_{\alpha}^{\nu}\right\}-(\mu \leftrightarrow \nu)\right] .
\end{aligned}
$$

We notice that the Yang-Mills amplitude has no explicit dependence on the spacetime dimension, whereas some of the contributions to the axion amplitude above do. This means that terms involving the dimension of spacetime should cancel with each other. In addition, each diagram satisfies the Ward identity $k_{\mu} \tilde{J}^{\mu \nu}(k)=0$ and so does the total amplitude, collected in Eq. (B1) of Appendix B. The total amplitude is in agreement with the calculation in the string frame metric, presented in [20]. We find that it matches the double copy result, Eq. (45).

\section{Graviton radiation}

Similarly, at the next to leading order in perturbation, the energy-momentum pseudotensor receives contributions from Figs. 3(a)-3(d). The contribution from Figs. 3(a) and 3(b) come from deflections to the particle spin and trajectory due to the leading order fields. This comes out to be

$$
\begin{aligned}
\left.\tilde{T}^{\mu \nu}(k)\right|_{\text {Fig. } 3(\mathrm{~b}) ; \mathcal{O}\left(\eta^{2}, S^{1}\right)=} & \frac{1}{2} \sum_{\alpha} \int d \tau_{\alpha} e^{i k \cdot x_{\alpha}}\left[\frac{-i}{k \cdot v_{\alpha}}\left\{\frac{k \cdot \dot{v}_{\alpha}}{k \cdot v_{\alpha}} v_{\alpha}^{\mu} p_{\alpha}^{\nu}-\dot{v}_{\alpha}^{\mu} p_{\alpha}^{\nu}-v_{\alpha}^{\mu} \dot{p}_{\alpha}^{\nu}\right\}\right. \\
& \left.-\frac{k_{\rho}}{k \cdot v_{\alpha}}\left\{\frac{k \cdot \dot{v}_{\alpha}}{k \cdot v_{\alpha}} v_{\alpha}^{\mu} S_{\alpha}^{\nu \rho}-\dot{v}_{\alpha}^{\mu} S_{\alpha}^{\nu \rho}\right\}+(\mu \leftrightarrow \nu)\right]\left.\right|_{\mathcal{O}\left(\eta^{2}, S^{1}\right)} .
\end{aligned}
$$


These contributions are explicitly given by Eqs. (A5) and (A6).

There are two contributions from emission off bulk vertices with the particles not suffering any deflections. The first of these is from Fig. 3(c) with an intermediate graviton. This contributes

$$
\begin{aligned}
\left.\tilde{T}^{\mu \nu}(k)\right|_{\text {Fig. } 3(\mathrm{c}) ; \mathcal{O}\left(\eta^{2}, S^{1}\right)}= & \frac{i}{4 m_{\mathrm{Pl}}^{d-2}} \sum_{\substack{\alpha, \beta \\
\alpha \neq \beta}} \int d \mu_{\alpha \beta}(k) \frac{\ell_{\alpha}^{2}}{2}\left[\left(p_{\alpha} \cdot p_{\beta}\right)\left\{\left(S_{\alpha} \wedge p_{\beta}\right)^{\mu} \ell_{\beta}^{\nu}-\left(S_{\alpha} \wedge \ell_{\beta}\right)^{\mu} p_{\beta}^{\nu}\right\}\right. \\
& \left.-\left(k \cdot p_{\alpha}\right)\left(S_{\alpha} \wedge p_{\beta}\right)^{\mu} p_{\beta}^{\nu}+\frac{m_{\beta}^{2}}{d-2}\left(S_{\alpha} \wedge \ell_{\beta}\right)^{\mu} p_{\alpha}^{\nu}+(\mu \leftrightarrow \nu)\right] .
\end{aligned}
$$

The final contribution is from the graviton triple vertex diagram in Fig. 3(d). As in [14], in computing this contribution, we use the background field gauge three-point vertex, written, for example, in $[11,36]$. This gives

$$
\begin{aligned}
\left.\tilde{T}^{\mu \nu}(k)\right|_{\text {Fig. } 3(\mathrm{~d}) ; \mathcal{O}\left(\eta^{2}, S^{1}\right)}= & \frac{i}{4 m_{\mathrm{Pl}}^{d-2}} \sum_{\substack{\alpha, \beta \\
\alpha \neq \beta}} \int d \mu_{\alpha \beta}(k)\left[( p _ { \alpha } \cdot p _ { \beta } ) \left\{\left(\ell_{\alpha} \cdot \ell_{\beta}\right)\left(S_{\alpha} \wedge \ell_{\alpha}\right)^{\mu} p_{\beta}^{\nu}+\left(k \cdot p_{\beta}\right)\left(S_{\alpha} \wedge \ell_{\alpha}\right)^{\mu} \ell_{\alpha}^{\nu}\right.\right. \\
& \left.+\left(\ell_{\alpha} \wedge \ell_{\beta}\right)_{\alpha} p_{\beta}^{\mu} l_{\alpha}^{\nu}+\left(\ell_{\alpha} \wedge p_{\beta}\right)_{\alpha} \ell_{\alpha}^{\mu} \ell_{\alpha}^{\nu}-\frac{1}{2} \ell_{\alpha}^{2}\left(\ell_{\alpha} \wedge p_{\beta}\right)_{\alpha} \eta^{\mu \nu}\right\}-\left(k \cdot p_{\beta}\right)^{2}\left(S_{\alpha} \wedge \ell_{\alpha}\right)^{\mu} p_{\alpha}^{\nu} \\
& +\left(k \cdot p_{\alpha}\right)\left(k \cdot p_{\beta}\right)\left(S_{\alpha} \wedge \ell_{\alpha}\right)^{\mu} p_{\beta}^{\nu}+\left(k \cdot p_{\alpha}\right)\left(\ell_{\alpha} \wedge p_{\beta}\right)_{\alpha} p_{\beta}^{\mu} \ell_{\alpha}^{\nu}-\left(k \cdot p_{\beta}\right)\left(\ell_{\alpha} \wedge p_{\beta}\right)_{\alpha} p_{\alpha}^{\mu} \ell_{\alpha}^{\nu} \\
& -\left(k \cdot p_{\beta}\right)\left(\ell_{\alpha} \wedge \ell_{\beta}\right)_{\alpha} p_{\alpha}^{\mu} p_{\beta}^{\nu}+\left(k \cdot p_{\alpha}\right)\left(\ell_{\alpha} \wedge \ell_{\beta}\right)_{\alpha} p_{\beta}^{\mu} p_{\beta}^{\nu}-\left(\ell_{\alpha} \cdot \ell_{\beta}\right)\left(\ell_{\alpha} \wedge p_{\beta}\right)_{\alpha} p_{\alpha}^{\mu} p_{\beta}^{\nu} \\
& \left.+\frac{m_{\beta}^{2}}{d-2}\left(S_{\alpha} \wedge \ell_{\alpha}\right)^{\mu} p_{\alpha}^{\nu}+(\mu \leftrightarrow \nu)\right] .
\end{aligned}
$$

The total canonically normalized graviton amplitude is Eq. (B2) in Appendix B. It is easy to show that the on-shell difference between this and the double copy prediction in Eq. (47) is

$$
\begin{aligned}
\hat{\mathcal{A}}_{g}(k)-\left.\mathcal{A}_{g}(k)\right|_{\mathcal{O}\left(\eta^{3}, S^{1}\right)}= & \frac{i \epsilon_{\mu \nu}^{*}}{8 m_{\mathrm{Pl}}^{3(d-2) / 2}} \sum_{\substack{\alpha \beta \\
\alpha \neq \beta}} \int d \mu_{\alpha \beta}(k)\left[\frac { p _ { \alpha } \cdot p _ { \beta } } { 2 ( k \cdot p _ { \alpha } ) } \left\{-\frac{1}{2} \ell_{\alpha}^{2}\left(p_{\alpha} \cdot p_{\beta}\right)\left(S_{\alpha} \wedge k\right)^{\mu} k^{\nu}-\left(\ell_{\alpha} \cdot \ell_{\beta}\right)\left(\ell_{\alpha} \wedge p_{\beta}\right)_{\alpha} k^{u} p_{\alpha}^{\nu}\right.\right. \\
& \left.-\frac{1}{2} \ell_{\alpha}^{2}\left\{\left(\ell_{\beta} \wedge p_{\beta}\right)_{\alpha}-\left(\ell_{\beta} \wedge p_{\alpha}\right)_{\beta}\right\} k^{u} p_{\alpha}^{\nu}\right\}+\frac{1}{2}\left(p_{\alpha} \cdot p_{\beta}\right)\left\{\left(k \cdot p_{\beta}\right)\left(S_{\alpha} \wedge \ell_{\alpha}\right)^{\mu} k^{\nu}+\frac{1}{2} \ell_{\alpha}^{2}\left(S_{\alpha} \wedge p_{\beta}\right)^{\mu} k^{\nu}\right. \\
& \left.+\left(\ell_{\alpha} \wedge \ell_{\beta}\right)_{\alpha} k^{\nu} p_{\beta}^{\mu}-\frac{1}{2}\left(\ell_{\alpha} \wedge p_{\beta}\right)_{\alpha} k^{u} k^{\nu}+2\left(\ell_{\alpha} \wedge p_{\beta}\right)_{\alpha} \ell_{\alpha}^{\mu} k^{\nu}-\ell_{\alpha}^{2}\left(l_{\alpha} \wedge p_{\beta}\right)_{\alpha} \eta^{\mu \nu}\right\} \\
& \left.-\frac{1}{2}\left(\ell_{\alpha} \wedge p_{\beta}\right)_{\alpha}\left(\left(k \cdot p_{\beta}\right) p_{\alpha}^{\nu}-\left(k \cdot p_{\alpha}\right) p_{\beta}^{\nu}\right) k^{\mu}+(\mu \leftrightarrow \nu)\right]
\end{aligned}
$$

We then use the de Donder gauge condition to write this as

$$
\begin{aligned}
\hat{\mathcal{A}}_{g}(k)-\left.\mathcal{A}_{g}(k)\right|_{\mathcal{O}\left(\eta^{3}, S^{1}\right)}= & \frac{i \epsilon_{\mu \nu}^{*}}{8 m_{\mathrm{Pl}}^{3(d-2) / 2}} \sum_{\substack{\alpha, \beta \\
\alpha \neq \beta}} \int d \mu_{\alpha \beta}(k) \frac{\eta^{\mu \nu}}{4}\left[( p _ { \alpha } \cdot p _ { \beta } ) \left\{\left(-2 \ell_{\alpha}^{2}+2 k \cdot \ell_{\alpha}-\left(\ell_{\alpha} \cdot \ell_{\beta}\right)\right)\left(\ell_{\alpha} \wedge p_{\beta}\right)_{\alpha}\right.\right. \\
& \left.-\frac{1}{2} \ell_{\alpha}^{2}\left(\left(\ell_{\beta} \wedge p_{\beta}\right)_{\alpha}-\left(\ell_{\beta} \wedge p_{\alpha}\right)_{\beta}-\left(k \wedge p_{\beta}\right)_{\alpha}\right)+\left(k \cdot p_{\beta}\right)\left(\left(k \wedge \ell_{\alpha}\right)_{\alpha}+\left(\ell_{\alpha} \wedge \ell_{\beta}\right)_{\alpha}\right)\right\} \\
& +(\mu \leftrightarrow \nu)],
\end{aligned}
$$



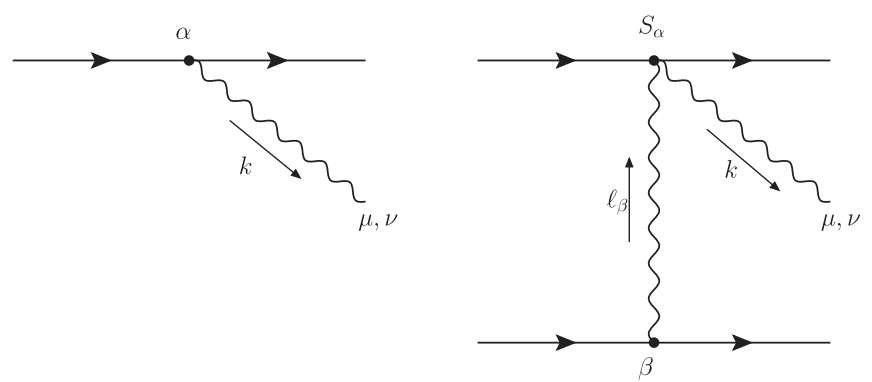

(a)

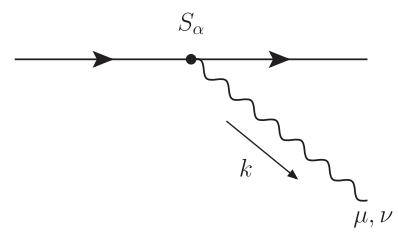

(b)

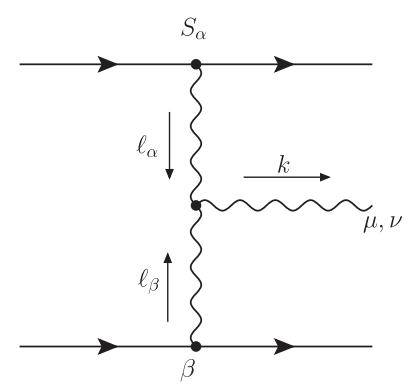

(d)

$$
\begin{aligned}
& \left.\tilde{J}(k)\right|_{\text {Fig. } 4(\mathrm{a}) ; \mathcal{O}\left(\eta^{2}, S^{1}\right)} \\
& =\sum_{\alpha} \int d \tau_{\alpha} e^{i k \cdot x_{\alpha}} \frac{i}{k \cdot v_{\alpha}} \\
& \quad \times\left.\left[\frac{k \cdot \dot{v}_{\alpha}}{k \cdot v_{\alpha}} v_{\alpha} \cdot p_{\alpha}-\dot{v}_{\alpha} \cdot p_{\alpha}-v_{\alpha} \cdot \dot{p}_{\alpha}\right]\right|_{\mathcal{O}\left(\eta^{2}, S^{1}\right)} .
\end{aligned}
$$

This expression can be computed using the leading order changes in momenta derived in Sec. IV B. The result is displayed as Eq. (A7) in Appendix A 2.

The other two contributions are calculated at zero deflections in the particle trajectories. Figure 4(b) involving an intermediate graviton contributes

$$
\begin{aligned}
& \left.\tilde{J}(k)\right|_{\text {Fig. 4(b);O }\left(\eta^{2}, S^{1}\right)} \\
& =-\frac{i}{2 m_{\mathrm{Pl}}^{d-2}} \sum_{\substack{\alpha, \beta \\
\alpha \neq \beta}} \int d \mu_{\alpha \beta}(k) \ell_{\alpha}^{2}\left(p_{\alpha} \cdot p_{\beta}\right)\left(\ell_{\beta} \wedge p_{\alpha}\right)_{\beta} .
\end{aligned}
$$

Finally, we have Fig. 4(c) involving the three-point graviton-dilaton vertex in the bulk, contributing

FIG. 3. Feynman diagrams that contribute to the energymomentum pseudotensor $\tilde{T}^{\mu \nu}(k)$ at $\mathcal{O}\left(\eta^{2}\right)$ and to linear order in spin. (a) Contribution from the spin-independent part due to the equations of motion. (b)-(d) Contributions from a single insertion of the spin-dependent piece of the point-particle energy-momentum pseudotensor.

which can be easily shown to vanish on shell. Thus, we have verified that the graviton amplitude is as predicted up to gauge terms.

\section{E. Dilaton radiation}

In this subsection, we calculate the leading order dilaton radiation amplitude. Figure 4(a) gives contributions to the dilaton amplitude from trajectory deflections,

$$
\begin{aligned}
& \left.\tilde{J}(k)\right|_{\text {Fig. } 4(\mathrm{c}) ; \mathcal{O}\left(\eta^{2}, S^{1}\right)} \\
& \quad=\frac{i}{2 m_{\mathrm{Pl}}^{d-2}} \sum_{\substack{\alpha, \beta \\
\alpha \neq \beta}} m_{\alpha}^{2} \int d \mu_{\alpha, \beta}(k)\left(k \cdot p_{\beta}\right)\left(\ell_{\alpha} \wedge \ell_{\beta}\right)_{\beta} .
\end{aligned}
$$

These individual contributions can be summed up to give the total dilaton amplitude, presented as Eq. (B3) in Appendix B. This agrees with the double copy prediction in Eq. (49).

\section{DISCUSSIONS AND CONCLUSIONS}

Lessons learned from the study of scattering amplitudes in quantum field theory and string theory are proving useful in the study of classical gravity. Some examples of this are

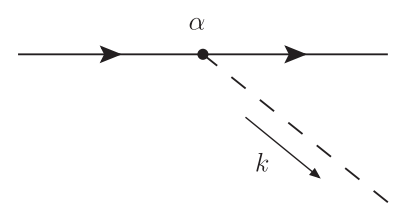

(a)

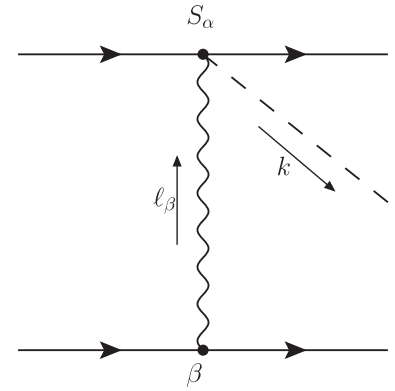

(b)

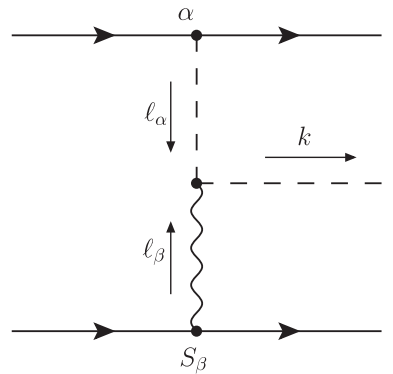

(c)

FIG. 4. Feynman diagrams contributing to the dilaton source $\tilde{J}(k)$ at $\mathcal{O}\left(\eta^{2}\right)$ and to linear order in spin. As earlier, the dashed lines and the wavy lines represent the dilaton and the graviton respectively. (a) Contribution to the spin-independent piece of $\tilde{J}(k)$ due to the equations of motion. (b),(c) Contributions from a single insertion of the spin dependent piece. 
the use of on-shell methods [37,38], leading singularities [39], and soft theorems [40]. In this paper, we continued exploring the idea of using the BCJ double copy to obtain perturbative classical gravitational radiation [14]. Specifically, we have established a complete correspondence between perturbative classical Yang-Mills and gravitational radiation, emitted by spinning sources, up to linear order in spin. Consistency with the double copy fixes the couplings of the spinning sources to the Yang-Mills field. The double copied field is decomposed into its antisymmetric, symmetric-traceless, and trace components. These are the predictions of the double copy for the axion, graviton, and dilaton radiation fields emitted by spinning sources in a gravitational theory. The double copy also fixes the gravitational action in the bulk to be the same as the low energy effective action of oriented closed strings in Eq. (6) and predicts a linear interaction of the spins with the axion in Eq. (7). We explicitly calculated the graviton, dilaton, and axion radiation amplitudes in this theory (see [41] for analogous pure gravity results), to linear order in spin, and showed that they are exactly as predicted from the double copy.

By including spin as a dynamical degree of freedom for our sources, we have brought the classical double copy closer to being useful for the computation of gravitational radiation from astrophysically relevant sources. Our calculation can be used to generate gravitational radiation from sources along arbitrary trajectories. Hence, they can be used to compute gravitational scattering as well as radiation from objects in bound orbits. However, the gravitational radiation so obtained is in a theory which includes the dilaton and the axion, in addition to the graviton. In order to arrive at the corresponding results in pure gravity, we need a systematic method that cancels out the effect of these extra degrees of freedom. For spinless sources, Ref. [42] proposed a way of removing the contribution of the dilaton at leading order in perturbation, by adding an appropriate ghost scalar to the Yang-Mills side, in a manner similar to [43]. Once we find a method of obtaining pure gravity results, it would be useful to write down an effective theory for the Yang-Mills sources that, upon double copying, directly reproduces the effective field theory in $[11,12]$, which treats the binary inspiral problem in a post-Newtonian expansion.

For $d=4$, the leading order metric sourced by a single, static particle at the origin, with angular momentum $J$ about the $z$-axis, has a spin-dependent piece given by $\left.h_{t \phi}\right|_{\mathcal{O}\left(J^{1}\right)}=-\frac{2 G_{N} J \sin ^{2} \theta}{r}$. We recall that the nonspinning part of the metric at this order in perturbation matches with the Schwarzschild solution [14]. At leading order in perturbation, there are no contributions from the dilaton or the axion to the metric. Hence, as expected, we find that the static, single particle limit of the metric matches the Kerr metric at this order. This indicates that the gravitational solutions are spinning black holes or naked singularities [42], albeit with dilatonic and axionic hair. It would be very interesting to see if they are related to black hole solutions in string theory.

In order to be relevant for gravitational wave detectors, the double copy correspondence needs to be extended to higher orders, both in the coupling strength and in spin. We would also need to include finite size corrections to the sources by including higher order operators on the worldline that are allowed by the symmetries. The correspondence can be extended to charged, spinning sources. A further simplification of the results in this paper can be achieved by finding the analogous biadjoint scalar theory $[15,44,45]$, the double copy of whose solution would produce the Yang-Mills radiation calculated here. A different approach to simplifying the perturbative expansion of the Einstein-Hilbert Lagrangian is by direct factorization of the action [46,47]. It would be interesting to see if it is related to the classical double copy as described here. Another direction for future research is to explore the class of solutions of Einstein's equations that can be generated by this correspondence. Finally, the theoretical significance of the classical double copy for Einstein's general relativity remains an exciting and open question.

\section{ACKNOWLEDGMENTS}

We gratefully acknowledge Walter Goldberger for suggesting this problem, for many valuable discussions and collaboration, and also for comments on this manuscript. S. P. also thanks Ghanashyam Date, Brian Henning, Dileep Jatkar, Alok Laddha, R. Loganayagam, Shiraz Minwalla, Ashoke Sen, and Junpu Wang for useful discussions, as well as Brian Henning for a careful reading of an earlier version of this manuscript.

\section{APPENDIX A: WORLDLINE GRAPHS}

In these appendixes, we collect some expressions that were omitted from the main body of this paper. This section has those contributions to the total amplitudes that are due to radiation coming directly off the worldline. The next two subsections present the relevant contributions in the gauge and gravitational theories, respectively.

\section{Gauge theory}

The relevant contributions are from Figs. 1(a) and 1(b) and are given by Eqs. (25a) and (25b). These can be written down explicitly as 


$$
\begin{aligned}
& (25 \mathrm{a})=i g_{s}^{2} \sum_{\substack{\alpha, \beta \\
\alpha \neq \beta}} \int d \mu_{\alpha \beta}(k)\left[\left[c_{\alpha}, c_{\beta}\right]^{a} \frac{\ell_{\alpha}^{2}}{k \cdot p_{\alpha}}\left\{\kappa_{\alpha}\left(\ell_{\beta} \wedge p_{\beta}\right)_{\alpha} p_{\alpha}^{\mu}-\kappa_{\beta}\left(\ell_{\beta} \wedge p_{\alpha}\right)_{\beta} p_{\alpha}^{\mu}\right\}\right. \\
& +\left(c_{\alpha} \cdot c_{\beta}\right) c_{\alpha}^{a}\left\{\left(1+\kappa_{\alpha}\right) \frac{\ell_{\alpha}^{2}}{p_{\alpha}^{2}}\left[\left(p_{\alpha} \cdot p_{\beta}\right)\left(S_{\alpha} \wedge \ell_{\beta}\right)^{\mu}-\left(k \cdot p_{\alpha}\right)\left(S_{\alpha} \wedge p_{\beta}\right)^{\mu}+\left(k \wedge p_{\beta}\right)_{\alpha} p_{\alpha}^{\mu}-\frac{p_{\alpha} \cdot p_{\beta}}{k \cdot p_{\alpha}}\left(\ell_{\alpha} \wedge \ell_{\beta}\right)_{\alpha} p_{\alpha}^{\mu}\right]\right. \\
& +\kappa_{\beta} \frac{\ell_{\alpha}^{2}}{k \cdot p_{\alpha}}\left[\left(k \cdot p_{\alpha}\right)\left(S_{\beta} \wedge \ell_{\beta}\right)^{\mu}+\left(\ell_{\beta} \wedge \ell_{\alpha}\right)_{\beta} p_{\alpha}^{\mu}+\left(\ell_{\beta} \wedge p_{\alpha}\right)_{\beta}\left(\ell_{\beta}^{\mu}-\frac{k \cdot \ell_{\beta}}{k \cdot p_{\alpha}} p_{\alpha}^{\mu}\right)\right] \\
& \left.\left.-\kappa_{\alpha} \frac{\ell_{\alpha}^{2}}{k \cdot p_{\alpha}}\left[\left(\ell_{\beta} \wedge p_{\beta}\right)_{\alpha}\left(\ell_{\beta}^{\mu}-\frac{k \cdot \ell_{\beta}}{k \cdot p_{\alpha}} p_{\alpha}^{\mu}\right)\right]\right\}\right], \\
& (25 \mathrm{~b})=i g_{s}^{2} \sum_{\substack{\alpha, \beta \\
\alpha \neq \beta}} \int d \mu_{\alpha \beta}(k)\left[\left[c_{\alpha}, c_{\beta}\right]^{a} \frac{p_{\alpha} \cdot p_{\beta}}{k \cdot p_{\alpha}} \ell_{\alpha}^{2}\left(S_{\alpha} \wedge k\right)^{\mu}\right. \\
& +\left(c_{\alpha} \cdot c_{\beta}\right) c_{\alpha}^{a}\left\{\left(1+\kappa_{\alpha}\right) \frac{\ell_{\alpha}^{2}}{p_{\alpha}^{2}}\left[\left(p_{\alpha} \cdot p_{\beta}\right)\left(S_{\alpha} \wedge \ell_{\beta}\right)^{\mu}-\left(k \cdot p_{\alpha}\right)\left(S_{\alpha} \wedge p_{\beta}\right)^{\mu}+\left(k \wedge p_{\beta}\right)_{\alpha} p_{\alpha}^{\mu}-\frac{p_{\alpha} \cdot p_{\beta}}{k \cdot p_{\alpha}}\left(\ell_{\alpha} \wedge \ell_{\beta}\right)_{\alpha} p_{\alpha}^{\mu}\right]\right. \\
& +\kappa_{\alpha} \frac{\ell_{\alpha}^{2}}{k \cdot p_{\alpha}}\left[\left(\ell_{\alpha} \wedge \ell_{\beta}\right)_{\alpha} p_{\beta}^{\mu}-\left(k \wedge p_{\beta}\right)_{\alpha} \ell_{\beta}^{\mu}-\left(k \cdot p_{\beta}\right)\left(S_{\alpha} \wedge \ell_{\beta}\right)^{\mu}+\left(k \cdot \ell_{\beta}\right)\left(S_{\alpha} \wedge p_{\beta}\right)^{\mu}\right] \\
& \left.\left.+\frac{\ell_{\alpha}^{2}}{k \cdot p_{\alpha}}\left[\frac{p_{\alpha} \cdot p_{\beta}}{k \cdot p_{\alpha}}\left(k \cdot \ell_{\beta}\right)-\left(k \cdot p_{\beta}\right)\right]\left(S_{\alpha} \wedge k\right)^{\mu}\right\}\right]
\end{aligned}
$$

\section{Gravitational theory}

First, we look at the axion current. This receives contributions from the worldline diagram in Fig. 2(a), given by Eqs. (86a) and (86b). These compute to

$$
\begin{gathered}
(86 \mathrm{a})=-\frac{i}{16 m_{\mathrm{Pl}}^{d-2}} \sum_{\substack{\alpha, \beta \\
\alpha \neq \beta}} \int d \mu_{\alpha \beta}(k) \ell_{\alpha}^{2}\left[-\frac{\left(p_{\alpha} \cdot p_{\beta}\right)^{2}\left(k \cdot \ell_{\beta}\right)}{\left(k \cdot p_{\alpha}\right)^{2}}\left(S_{\alpha} \wedge k\right)^{\mu} p_{\alpha}^{\nu}\right. \\
\left.+\frac{p_{\alpha} \cdot p_{\beta}}{k \cdot p_{\alpha}}\left\{\left(p_{\alpha} \cdot p_{\beta}\right) \ell_{\beta}^{\nu}-2\left(k \cdot p_{\alpha}\right) p_{\beta}^{\nu}+2\left(k \cdot p_{\beta}\right) p_{\alpha}^{\nu}\right\}\left(S_{\alpha} \wedge k\right)^{\mu}-(\mu \leftrightarrow \nu)\right], \\
(86 \mathrm{~b})=-\frac{i}{16 m_{\mathrm{Pl}}^{d-2}} \sum_{\substack{\alpha, \beta \\
\alpha \neq \beta}} \int d \mu_{\alpha \beta}(k) \ell_{\alpha}^{2}\left[\frac { p _ { \alpha } \cdot p _ { \beta } } { k \cdot p _ { \alpha } } \left\{\left(k \cdot \ell_{\beta}\right)\left(S_{\alpha} \wedge p_{\beta}\right)^{\mu} p_{\beta}^{\nu}-\left(k \cdot p_{\beta}\right)\left(S_{\alpha} \wedge \ell_{\beta}\right)^{\mu} p_{\beta}^{\nu}-\left(k \wedge \ell_{\beta}\right)_{\alpha} p_{\alpha}^{\mu} p_{\beta}^{\nu}\right.\right. \\
\left.+\left(k \wedge p_{\beta}\right)_{\alpha} p_{\alpha}^{\mu} \ell_{\beta}^{\nu}\right\}+\left(p_{\alpha} \cdot p_{\beta}\right)\left\{\left(S_{\alpha} \wedge \ell_{\beta}\right)^{\mu} p_{\beta}^{\nu}-\left(S_{\alpha} \wedge p_{\beta}\right)^{\mu} \ell_{\beta}^{\nu}\right\}+\left(k \cdot p_{\alpha}\right)\left(S_{\alpha} \wedge p_{\beta}\right)^{\mu} p_{\beta}^{\nu} \\
\left.-\left(k \cdot p_{\beta}\right)\left(S_{\alpha} \wedge p_{\beta}\right)^{\mu} p_{\alpha}^{\nu}-\left(k \wedge p_{\beta}\right)_{\alpha} p_{\alpha}^{\mu} p_{\beta}^{\nu}+\frac{p_{\beta}^{2}}{d-2}\left\{2\left(S_{\alpha} \wedge k\right)^{\mu}-\left(k \cdot p_{\alpha}\right) S_{\alpha}^{\mu \nu}\right\}-(\mu \leftrightarrow \nu)\right] .
\end{gathered}
$$

Next, we move on to graviton radiation. Worldline contributions to the energy-momentum pseudotensor are from Figs. 3(a) and 3(b). The corresponding expressions in Eqs. (90a) and (90b) are, respectively, given by

$$
\begin{aligned}
(90 \mathrm{a})= & \frac{i}{4 m_{\mathrm{Pl}}^{d-2}} \sum_{\substack{\alpha, \beta \\
\alpha \neq \beta}} \int d \mu_{\alpha \beta}(k) \ell_{\alpha}^{2}\left[\frac{\left(p_{\alpha} \cdot p_{\beta}\right)\left(k \cdot \ell_{\beta}\right)}{2\left(k \cdot p_{\alpha}\right)^{2}}\left\{\left(\ell_{\beta} \wedge p_{\beta}\right)_{\alpha}-\left(\ell_{\beta} \wedge p_{\alpha}\right)_{\beta}\right\} p_{\alpha}^{\mu} p_{\alpha}^{\nu}\right. \\
& -\frac{p_{\alpha} \cdot p_{\beta}}{k \cdot p_{\alpha}}\left\{\left(\left(\ell_{\beta} \wedge p_{\beta}\right)_{\alpha}-\left(\ell_{\beta} \wedge p_{\alpha}\right)_{\beta}\right) p_{\alpha}^{\mu} \ell_{\beta}^{\nu}+\frac{1}{2}\left(\ell_{\alpha} \wedge \ell_{\beta}\right)_{\beta} p_{\alpha}^{\mu} p_{\alpha}^{\nu}\right\}-\frac{k \cdot p_{\beta}}{2\left(k \cdot p_{\alpha}\right)}\left\{\left(\ell_{\beta} \wedge p_{\beta}\right)_{\alpha}-\left(\ell_{\beta} \wedge p_{\alpha}\right)_{\beta}\right\} p_{\alpha}^{\mu} p_{\alpha}^{\nu} \\
& \left.+\left(p_{\alpha} \cdot p_{\beta}\right)\left(S_{\beta} \wedge \ell_{\beta}\right)^{\mu} p_{\alpha}^{\nu}+\left\{\left(\ell_{\beta} \wedge p_{\beta}\right)_{\alpha}-\left(\ell_{\beta} \wedge p_{\alpha}\right)_{\beta}\right\} p_{\alpha}^{\mu} p_{\beta}^{\nu}+\frac{m_{\beta}^{2}}{2(d-2)}\left(S_{\alpha} \wedge \ell_{\beta}\right)^{\mu} p_{\alpha}^{\nu}+(\mu \leftrightarrow \nu)\right],
\end{aligned}
$$




$$
\begin{aligned}
(90 \mathrm{~b})= & \frac{i}{4 m_{\mathrm{Pl}}^{d-2}} \sum_{\substack{\alpha, \beta \\
\alpha \neq \beta}} \int d \mu_{\alpha \beta}(k) \ell_{\alpha}^{2}\left[\frac{\left(p_{\alpha} \cdot p_{\beta}\right)^{2}\left(k \cdot \ell_{\beta}\right)}{2\left(k \cdot p_{\alpha}\right)^{2}}\left(S_{\alpha} \wedge k\right)^{\mu} p_{\alpha}^{\nu}-\frac{\left(p_{\alpha} \cdot p_{\beta}\right)^{2}}{2\left(k \cdot p_{\alpha}\right)}\left(S_{\alpha} \wedge k\right)^{\mu} \ell_{\beta}^{\nu}\right. \\
& -\frac{p_{\alpha} \cdot p_{\beta}}{2\left(k \cdot p_{\alpha}\right)}\left\{\left(k \cdot p_{\beta}\right)\left(\left(S_{\alpha} \wedge k\right)^{\mu}+\left(S_{\alpha} \wedge \ell_{\alpha}\right)^{\mu}\right) p_{\alpha}^{\nu}+\left(k \cdot \ell_{\beta}\right)\left(S_{\alpha} \wedge p_{\beta}\right)^{\mu} p_{\alpha}^{\nu}+\left(\ell_{\alpha} \wedge \ell_{\beta}\right)_{\alpha} p_{\alpha}^{\mu} p_{\beta}^{\nu}-\left(k \wedge p_{\beta}\right)_{\alpha} p_{\alpha}^{\mu} \ell_{\beta}^{\nu}\right\} \\
& \left.+\left(p_{\alpha} \cdot p_{\beta}\right)\left(S_{\alpha} \wedge k\right)^{\mu} p_{\beta}^{\nu}+\frac{1}{2}\left(k \cdot p_{\beta}\right)\left(S_{\alpha} \wedge p_{\beta}\right)^{\mu} p_{\alpha}^{\nu}-\frac{1}{2}\left(k \wedge p_{\beta}\right)_{\alpha} p_{\alpha}^{\mu} p_{\beta}^{\nu}-\frac{m_{\beta}^{2}}{d-2}\left(S_{\alpha} \wedge k\right)^{\mu} p_{\alpha}^{\nu}+(\mu \leftrightarrow \nu)\right] .
\end{aligned}
$$

Finally, the dilaton worldline graph in Fig. 4(a) gives rise to the contribution in Eq. (95) and can be computed explicitly to be

$$
\begin{aligned}
(95)= & \frac{i}{4 m_{\mathrm{Pl}}^{d-2}} \sum_{\substack{\alpha, \beta \\
\alpha \neq \beta}} \int d \mu_{\alpha \beta}(k) \ell_{\alpha}^{2}\left[\frac{p_{\alpha} \cdot p_{\beta}}{\left(k \cdot p_{\alpha}\right)^{2}} p_{\alpha}^{2}\left(k \cdot \ell_{\beta}\right)\left\{\left(\ell_{\beta} \wedge p_{\alpha}\right)_{\beta}-\left(\ell_{\beta} \wedge p_{\beta}\right)_{\alpha}\right\}\right. \\
& \left.+\frac{p_{\alpha} \cdot p_{\beta}}{k \cdot p_{\alpha}} p_{\alpha}^{2}\left(\ell_{\alpha} \wedge \ell_{\beta}\right)_{\beta}+\frac{k \cdot p_{\beta}}{k \cdot p_{\alpha}} p_{\alpha}^{2}\left\{\left(\ell_{\beta} \wedge p_{\beta}\right)_{\alpha}-\left(\ell_{\beta} \wedge p_{\alpha}\right)_{\beta}\right\}+2\left(p_{\alpha} \cdot p_{\beta}\right)\left(\ell_{\beta} \wedge p_{\alpha}\right)_{\beta}\right]
\end{aligned}
$$

\section{APPENDIX B: TOTAL AMPLITUDES IN THE GRAVITATIONAL THEORY}

In Sec. IV, we computed all the contributions to the leading order radiation amplitudes on the gravitational side. Here, we assemble these contributions and write down the final expressions for these amplitudes.

First, the axion amplitude is given by

$$
\begin{aligned}
& \left.\mathcal{A}_{B}(k)\right|_{\mathcal{O}\left(\eta^{3}, S^{1}\right)}=-\frac{i a_{\mu \nu}^{*}}{16 m_{\mathrm{Pl}}^{3(d-2) / 2}} \sum_{\substack{\alpha, \beta \\
\alpha \neq \beta}} \int d \mu_{\alpha \beta}(k)\left[-\frac{\left(p_{\alpha} \cdot p_{\beta}\right)^{2}\left(k \cdot \ell_{\beta}\right) \ell_{\alpha}^{2}}{\left(k \cdot p_{\alpha}\right)^{2}}\left(S_{\alpha} \wedge k\right)^{\mu} p_{\alpha}^{\nu}+\frac{\left(p_{\alpha} \cdot p_{\beta}\right) \ell_{\alpha}^{2}}{k \cdot p_{\alpha}}\left\{\left(\left(p_{\alpha} \cdot p_{\beta}\right) \ell_{\beta}^{\nu}\right.\right.\right. \\
& \left.-2\left(k \cdot p_{\alpha}\right) p_{\beta}^{\nu}+2\left(k \cdot p_{\beta}\right) p_{\alpha}^{\nu}\right)\left(S_{\alpha} \wedge k\right)^{\mu}+\left(k \cdot \ell_{\beta}\right)\left(S_{\alpha} \wedge p_{\beta}\right)^{\mu} p_{\beta}^{\nu}-\left(k \cdot p_{\beta}\right)\left(S_{\alpha} \wedge \ell_{\beta}\right)^{\mu} p_{\beta}^{\nu}-\left(k \wedge \ell_{\beta}\right)_{\alpha} p_{\alpha}^{\mu} p_{\beta}^{\nu} \\
& \left.+\left(k \wedge p_{\beta}\right)_{\alpha} p_{\alpha}^{\mu} \ell_{\beta}^{\nu}\right\}+\left(p_{\alpha} \cdot p_{\beta}\right)\left\{\ell_{\alpha}^{2}\left(S_{\alpha} \wedge \ell_{\beta}\right)^{\mu} p_{\beta}^{\nu}-\ell_{\alpha}^{2}\left(S_{\alpha} \wedge p_{\beta}\right)^{\mu} \ell_{\beta}^{\nu}+2\left(k \cdot \ell_{\alpha}\right)\left(S_{\alpha} \wedge \ell_{\alpha}\right)^{\mu} p_{\beta}^{\nu}\right. \\
& \left.-2\left(k \cdot p_{\beta}\right)\left(S_{\alpha} \wedge \ell_{\alpha}\right)^{\mu} \ell_{\alpha}^{\nu}-2\left(k \wedge \ell_{\alpha}\right)_{\alpha} l_{\alpha}^{\mu} p_{\beta}^{\nu}\right\}+\ell_{\alpha}^{2}\left(k \cdot p_{\alpha}\right)\left(S_{\alpha} \wedge p_{\beta}\right)^{\mu} p_{\beta}^{\nu}-\ell_{\alpha}^{2}\left(k \cdot p_{\beta}\right)\left(S_{\alpha} \wedge p_{\beta}\right)^{\mu} p_{\alpha}^{\nu} \\
& -2\left(k \cdot p_{\alpha}\right)\left(k \cdot p_{\beta}\right)\left(S_{\alpha} \wedge \ell_{\alpha}\right)^{\mu} p_{\beta}^{\nu}+2\left(k \cdot p_{\beta}\right)^{2}\left(S_{\alpha} \wedge \ell_{\alpha}\right)^{\mu} p_{\alpha}^{\nu}-\ell_{\alpha}^{2}\left(k \wedge p_{\beta}\right)_{\alpha} p_{\alpha}^{\mu} p_{\beta}^{\nu}+2\left(k \cdot p_{\beta}\right)\left(k \wedge \ell_{\alpha}\right)_{\alpha} p_{\alpha}^{\mu} p_{\beta}^{\nu} \\
& \left.+2\left(k \cdot p_{\beta}\right)\left(\ell_{\alpha} \wedge p_{\beta}\right)_{\alpha} \ell_{\alpha}^{\mu} p_{\alpha}^{\nu}-2\left(k \cdot p_{\alpha}\right)\left(\ell_{\alpha} \wedge p_{\beta}\right)_{\alpha} \ell_{\alpha}^{\mu} p_{\beta}^{\nu}+2\left(k \cdot \ell_{\alpha}\right)\left(\ell_{\alpha} \wedge p_{\beta}\right)_{\alpha} p_{\alpha}^{\mu} p_{\beta}^{\nu}-(\mu \leftrightarrow \nu)\right] \text {. }
\end{aligned}
$$

The leading order graviton amplitude is given by

$$
\begin{aligned}
& \left.\mathcal{A}_{g}(k)\right|_{\mathcal{O}\left(\eta^{3}, S^{1}\right)}=-\frac{i \epsilon_{\mu \nu}^{*}}{8 m_{\mathrm{Pl}}^{3(d-2) / 2}} \sum_{\substack{\alpha, \beta \\
\alpha \neq \beta}} \int d \mu_{\alpha \beta}(k)\left[\frac { ( p _ { \alpha } \cdot p _ { \beta } ) ( k \cdot \ell _ { \beta } ) \ell _ { \alpha } ^ { 2 } } { 2 ( k \cdot p _ { \alpha } ) ^ { 2 } } \left\{\left(\left(\ell_{\beta} \wedge p_{\beta}\right)_{\alpha}-\left(\ell_{\beta} \wedge p_{\alpha}\right)_{\beta}\right) p_{\alpha}^{\mu} p_{\alpha}^{\nu}\right.\right. \\
& \left.+\left(p_{\alpha} \cdot p_{\beta}\right)\left(S_{\alpha} \wedge k\right)^{\mu} p_{\alpha}^{\nu}\right\}-\frac{\left(p_{\alpha} \cdot p_{\beta}\right) \ell_{\alpha}^{2}}{k \cdot p_{\alpha}}\left\{\frac{1}{2}\left(p_{\alpha} \cdot p_{\beta}\right)\left(S_{\alpha} \wedge k\right)^{\mu} \ell_{\alpha}^{\nu}+\frac{1}{2}\left(k \cdot p_{\beta}\right)\left(\left(S_{\alpha} \wedge k\right)^{\mu}+\left(S_{\alpha} \wedge \ell_{\alpha}\right)^{\mu}\right) p_{\alpha}^{\nu}\right. \\
& +\frac{1}{2}\left(k \cdot \ell_{\beta}\right)\left(S_{\alpha} \wedge p_{\beta}\right)^{\mu} p_{\alpha}^{\nu}+\frac{1}{2}\left(\ell_{\alpha} \wedge \ell_{\beta}\right)_{\beta} p_{\alpha}^{\mu} p_{\alpha}^{\nu}+\frac{1}{2}\left(\ell_{\alpha} \wedge \ell_{\beta}\right)_{\alpha} p_{\alpha}^{\mu} p_{\beta}^{\nu}+\left(\left(\ell_{\beta} \wedge p_{\beta}\right)_{\alpha}-\left(\ell_{\beta} \wedge p_{\alpha}\right)_{\beta}\right. \\
& \left.\left.-\frac{1}{2}\left(k \wedge p_{\beta}\right)_{\alpha}\right) p_{\alpha}^{\mu} \ell_{\beta}^{\nu}\right\}-\frac{\left(k \cdot p_{\beta}\right) \ell_{\alpha}^{2}}{2\left(k \cdot p_{\alpha}\right)}\left\{\left(\ell_{\beta} \wedge p_{\beta}\right)_{\alpha}-\left(\ell_{\beta} \wedge p_{\alpha}\right)_{\beta}\right\} p_{\alpha}^{\mu} p_{\alpha}^{\nu}+\left(p_{\alpha} \cdot p_{\beta}\right)\left\{\frac{\ell_{\alpha}^{2}}{2}\left(S_{\alpha} \wedge \ell_{\beta}\right)^{\mu} p_{\beta}^{\nu}\right. \\
& -\left(\ell_{\alpha} \cdot \ell_{\beta}\right)\left(S_{\alpha} \wedge \ell_{\alpha}\right)^{\mu} p_{\beta}^{\nu}+\left(k \cdot p_{\beta}\right)\left(S_{\alpha} \wedge \ell_{\alpha}\right)^{\mu} \ell_{\alpha}^{\nu}+\frac{1}{2} \ell_{\alpha}^{2}\left(S_{\alpha} \wedge p_{\beta}\right)^{\mu} \ell_{\beta}^{\nu}+\left(\ell_{\alpha} \wedge \ell_{\beta}\right)_{\alpha} p_{\beta}^{\mu} \ell_{\alpha}^{\nu}+\left(\ell_{\alpha} \wedge p_{\beta}\right)_{\alpha} \ell_{\alpha}^{\mu} \ell_{\alpha}^{\nu} \\
& \left.-\frac{\ell_{\alpha}^{2}}{2}\left(\ell_{\alpha} \wedge p_{\beta}\right)_{\alpha} \eta^{\mu \nu}\right\}+\frac{1}{2}\left\{\ell_{\alpha}^{2}\left(\ell_{\beta} \wedge p_{\beta}\right)_{\alpha}-\ell_{\beta}^{2}\left(\ell_{\alpha} \wedge p_{\beta}\right)_{\alpha}\right\} p_{\alpha}^{\mu} p_{\beta}^{\nu}+\left\{\left(k \cdot p_{\beta}\right)\left(S_{\alpha} \wedge \ell_{\alpha}\right)^{\mu}-\frac{\ell_{\alpha}^{2}}{2}\left(S_{\alpha} \wedge p_{\beta}\right)^{\mu}\right. \\
& \left.\left.+\left(\ell_{\alpha} \wedge p_{\beta}\right)_{\alpha} l_{\alpha}^{\mu}+\left(\ell_{\alpha} \wedge \ell_{\beta}\right)_{\alpha} p_{\beta}^{\mu}\right\}\left(\left(k \cdot p_{\alpha}\right) p_{\beta}^{\nu}-\left(k \cdot p_{\beta}\right) p_{\alpha}^{\nu}\right)+(\mu \leftrightarrow \nu)\right]
\end{aligned}
$$


and finally, we have the leading order dilaton amplitude,

$$
\begin{aligned}
\left.\mathcal{A}_{s}(k)\right|_{\mathcal{O}\left(\eta^{3}, S^{1}\right)}= & -\frac{i}{8 m_{\mathrm{Pl}}^{3(d-2) / 2}(d-2)^{1 / 2}} \sum_{\substack{\alpha, \beta \\
\alpha \neq \beta}} \int d \mu_{\alpha \beta}(k) p_{\alpha}^{2}\left[\frac{p_{\alpha} \cdot p_{\beta}}{\left(k \cdot p_{\alpha}\right)^{2}} \ell_{\alpha}^{2}\left(k \cdot \ell_{\beta}\right)\left\{\left(\ell_{\beta} \wedge p_{\alpha}\right)_{\beta}-\left(\ell_{\beta} \wedge p_{\beta}\right)_{\alpha}\right\}\right. \\
& \left.+\frac{k \cdot p_{\beta}}{k \cdot p_{\alpha}} \ell_{\alpha}^{2}\left\{\left(\ell_{\beta} \wedge p_{\beta}\right)_{\alpha}-\left(\ell_{\beta} \wedge p_{\alpha}\right)_{\beta}\right\}+\frac{p_{\alpha} \cdot p_{\beta}}{k \cdot p_{\alpha}} \ell_{\alpha}^{2}\left(\ell_{\alpha} \wedge \ell_{\beta}\right)_{\beta}+2\left(k \cdot p_{\beta}\right)\left(\ell_{\alpha} \wedge \ell_{\beta}\right)_{\beta}\right] .
\end{aligned}
$$

[1] Z. Bern, J. J. M. Carrasco, and H. Johansson, Phys. Rev. D 78, 085011 (2008).

[2] Z. Bern, J. J. M. Carrasco, and H. Johansson, Phys. Rev. Lett. 105, 061602 (2010).

[3] Z. Bern, T. Dennen, Y.t. Huang, and M. Kiermaier, Phys. Rev. D 82, 065003 (2010).

[4] H. Kawai, D. C. Lewellen, and S. H. H. Tye, Nucl. Phys. B269, 1 (1986).

[5] Z. Bern, J. J. M. Carrasco, L. J. Dixon, H. Johansson, and R. Roiban, Phys. Rev. D 85, 105014 (2012); Z. Bern, C. Boucher-Veronneau, and H. Johansson, Phys. Rev. D 84, 105035 (2011); C. Boucher-Veronneau and L. J. Dixon, J. High Energy Phys. 12 (2011) 046; J. J. M. Carrasco and H. Johansson, Phys. Rev. D 85, 025006 (2012); Z. Bern, S. Davies, T. Dennen, Y. T. Huang, and J. Nohle, Phys. Rev. D 92, 045041 (2015); J. J. M. Carrasco, M. Chiodaroli, M. Gunaydin, and R. Roiban, J. High Energy Phys. 03 (2013) 056; M. Chiodaroli, M. Gunaydin, H. Johansson, and R. Roiban, J. High Energy Phys. 01 (2015) 081; Phys. Rev. Lett. 117, 011603 (2016); Z. Bern, J. J. M. Carrasco, W. M. Chen, H. Johansson, R. Roiban, and M. Zeng, Phys. Rev. D 96, 126012 (2017); Z. Bern, J. J. Carrasco, W. M. Chen, H. Johansson, and R. Roiban, Phys. Rev. Lett. 118, 181602 (2017).

[6] J. J. M. Carrasco, arXiv:1506.00974.

[7] R. Monteiro, D. O'Connell, and C. D. White, J. High Energy Phys. 12 (2014) 056.

[8] A. Luna, R. Monteiro, D. O'Connell, and C. D. White, Phys. Lett. B 750, 272 (2015).

[9] A. Luna, R. Monteiro, I. Nicholson, D. O'Connell, and C. D. White, J. High Energy Phys. 06 (2016) 023.

[10] A. K. Ridgway and M. B. Wise, Phys. Rev. D 94, 044023 (2016); G. L. Cardoso, S. Nagy, and S. Nampuri, J. High Energy Phys. 10 (2016) 127; G. Cardoso, S. Nagy, and S. Nampuri, J. High Energy Phys. 04 (2017) 037; Y. Z. Chu, Class. Quantum Grav. 34, 194001 (2017); T. Adamo, E. Casali, L. Mason, and S. Nekovar, Class. Quantum Grav. 35, 015004 (2018); N. Bahjat-Abbas, A. Luna, and C. D. White, J. High Energy Phys. 12 (2017) 004; M. Carrillo-Gonzalez, R. Penco, and M. Trodden, J. High Energy Phys. 04 (2018) 028.

[11] W. D. Goldberger and I.Z. Rothstein, Phys. Rev. D 73, 104029 (2006).

[12] R. A. Porto, Phys. Rev. D 73, 104031 (2006).

[13] R. A. Porto, Phys. Rep. 633, 1 (2016).
[14] W. D. Goldberger and A. K. Ridgway, Phys. Rev. D 95, 125010 (2017).

[15] W. D. Goldberger, S. G. Prabhu, and J. O. Thompson, Phys. Rev. D 96, 065009 (2017).

[16] W. D. Goldberger and A. K. Ridgway, Phys. Rev. D 97, 085019 (2018).

[17] B. P. Abbott et al. (LIGO Scientific and Virgo Collaborations), Phys. Rev. Lett. 119, 161101 (2017).

[18] D. Chester, Phys. Rev. D 97, 084025 (2018).

[19] A. Luna, R. Monteiro, I. Nicholson, A. Ochirov, D. O'Connell, N. Westerberg, and C. D. White, J. High Energy Phys. 04 (2017) 069.

[20] W. D. Goldberger, J. Li, and S. G. Prabhu, preceding paper, Phys. Rev. D 97, 105018 (2018).

[21] M. Mathisson, Acta Phys. Pol. 6, 163 (1937).

[22] A. Papapetrou, Proc. R. Soc. A 209, 248 (1951).

[23] W. G. Dixon, Proc. R. Soc. A 314, 499 (1970).

[24] A. J. Hanson and T. Regge, Ann. Phys. (N.Y.) 87, 498 (1974).

[25] I. Bailey and W. Israel, Commun. Math. Phys. 42, 65 (1975).

[26] R. A. Porto and I. Z. Rothstein, Phys. Rev. Lett. 97, 021101 (2006); Phys. Rev. D 78, 044012 (2008); 81, 029904(E) (2010); 78, 044013 (2008); 81, 029905(E) (2010).

[27] P. Sikivie and N. Weiss, Phys. Rev. D 18, 3809 (1978).

[28] M. Kalb and P. Ramond, Phys. Rev. D 9, 2273 (1974).

[29] M. H. L. Pryce, Proc. R. Soc. A 195, 62 (1948).

[30] S. Ferrara, M. Porrati, and V. L. Telegdi, Phys. Rev. D 46, 3529 (1992).

[31] B. R. Holstein, Phys. Rev. D 74, 085002 (2006).

[32] Z. Bern, S. Davies, and J. Nohle, Phys. Rev. D 93, 105015 (2016).

[33] J. Scherk and J. H. Schwarz, Phys. Lett. 52B, 347 (1974).

[34] D. J. Gross and J. H. Sloan, Nucl. Phys. B291, 41 (1987).

[35] B. S. DeWitt, Phys. Rev. 162, 1195 (1967); L. F. Abbott, Nucl. Phys. B185, 189 (1981).

[36] J. F. Donoghue, Phys. Rev. D 50, 3874 (1994).

[37] N.E. J. Bjerrum-Bohr, J.F. Donoghue, and P. Vanhove, J. High Energy Phys. 02 (2014) 111.

[38] D. Neill and I. Z. Rothstein, Nucl. Phys. B877, 177 (2013).

[39] F. Cachazo and A. Guevara, arXiv:1705.10262; A. Guevara, arXiv:1706.02314.

[40] A. Laddha and A. Sen, arXiv:1801.07719.

[41] D. Bini, A. Geralico, and J. Vines, Phys. Rev. D 96, 084044 (2017); J. Vines, Class. Quantum Grav. 35, 084002 (2018). 
[42] A. Luna, I. Nicholson, D. O'Connell, and C. D. White, J. High Energy Phys. 03 (2018) 044.

[43] H. Johansson and A. Ochirov, J. High Energy Phys. 11 (2015) 046.

[44] C. D. White, Phys. Lett. B 763, 365 (2016); P. J. De Smet and C. D. White, Phys. Lett. B 775, 163 (2017).
[45] F. Cachazo, S. He, and E. Y. Yuan, J. High Energy Phys. 07 (2014) 033.

[46] Z. Bern and A. K. Grant, Phys. Lett. B 457, 23 (1999).

[47] C. Cheung and G. N. Remmen, J. High Energy Phys. 01 (2017) 104; 09 (2017) 002. 